OPEN ACCESS

Edited by:

Hans-Peter Hartung,

Heinrich Heine University of

Düsseldorf, Germany

Reviewed by:

Elena Tchetina,

Nasonova Research Institute

of Rheumatology, Russia

Hyun Sook Hong,

Kyung Hee University, South Korea

*Correspondence:

Susanne Grässe

susanne.graessel@ukr.de

Specialty section:

This article was submitted to

Multiple Sclerosis and

Neuroimmunology,

a section of the journal

Frontiers in Immunology

Received: 09 June 2021 Accepted: 09 August 2021 Published: 27 August 2021

Citation:

Stöckl S, Eitner A, Bauer RJ, König M, Johnstone B and Grässel S (2021) Substance $P$ and Alpha-Calcitonin Gene-Related Peptide Differentially Affect Human Osteoarthritic and Healthy Chondrocytes.

Front. Immunol. 12:722884. doi: 10.3389/fimmu.2021.722884

\section{Substance P and Alpha-Calcitonin Gene-Related Peptide Differentially Affect Human Osteoarthritic and Healthy Chondrocytes}

\author{
Sabine Stöckl ${ }^{1}$, Annett Eitner ${ }^{2,3}$, Richard J. Bauer ${ }^{4}$, Matthias König ${ }^{5}$, Brian Johnstone ${ }^{6}$ \\ and Susanne Grässel ${ }^{1 *}$ \\ ${ }^{1}$ Department of Orthopaedic Surgery, Experimental Orthopaedics, Center for Medical Biotechnology, University of \\ Regensburg, Regensburg, Germany, ${ }^{2}$ Department of Trauma, Hand and Reconstructive Surgery, Experimental Trauma \\ Surgery, Jena University Hospital, Friedrich-Schiller-University Jena, Jena, Germany, ${ }^{3}$ Department of Physiology, Jena \\ University Hospital, Friedrich Schiller University Jena, Jena, Germany, ${ }^{4}$ Department of Oral and Maxillofacial Surgery, Center \\ for Medical Biotechnology, University Hospital Regensburg, Regensburg, Germany, ${ }^{5}$ Department of Orthopedics, University \\ Medical Center Regensburg, Asklepios Klinikum Bad Abbach, Bad Abbach, Germany, ${ }^{6}$ Department of Orthopaedics and \\ Rehabilitation, Oregon Health \& Science University, Portland, OR, United States
}

Osteoarthritis $(\mathrm{OA})$ is a degenerative joint disease that not only causes cartilage loss but also structural damage in all joint tissues. Joints are innervated by alpha-calcitonin generelated peptide ( $\alpha \mathrm{CGRP}$ ) and substance $\mathrm{P}(\mathrm{SP})$-positive sensory nerve fibers. Alteration of sensory joint innervation could be partly responsible for degenerative changes in joints that contribute to the development of OA. Therefore, our aim was to analyze and compare the molecular effects of SP and $\alpha$ CGRP on the metabolism of articular chondrocytes from OA patients and non-OA cartilage donors. We treated the cells with SP or $\alpha$ CGRP and analysed the influence of these neuropeptides on chondrocyte metabolism and modulation of signaling pathways. In chondrocytes from healthy cartilage, SP had minimal effects compared with its effects on OA chondrocytes, where it induced inflammatory mediators, inhibited chondrogenic markers and promoted apoptosis and senescence. Treatment with $\alpha$ CGRP also increased apoptosis and senescence and reduced chondrogenic marker expression in OA chondrocytes, but stimulated an anabolic and protective response in healthy chondrocytes. The catabolic influence of SP and $\alpha$ CGRP might be due to activation of ERK signaling that could be counteracted by an increased cAMP response. We suggest that a switch between the G-subunits of the corresponding receptors after binding their ligands SP or $\alpha$ CGRP plays a central role in mediating the observed effects of sensory neuropeptides on chondrocytes.

Keywords: osteoarthritis, SP, $\alpha$ CGRP, sensory nervous system, chondrocyte metabolism, ERK, cAMP, AKT

\section{INTRODUCTION}

Although osteoarthritis $(\mathrm{OA})$ is the world's most common musculoskeletal disorder, research efforts have not yet been able to define its exact etiology $(1,2)$. Changes in peripheral joint innervation may be partly responsible for degenerative alterations in joint tissues that contribute to the development of OA and to increased pain sensation. Sensory nerve fibers, releasing substance $\mathrm{P}$ 
(SP) and alpha-calcitonin gene-related peptide ( $\alpha$ CGRP), innervate synovium, trabecular and subchondral bone, bone marrow, periosteum and fracture callus. In addition to their classical neurological features, trophic effects that are critical for joint tissue and bone homeostasis under physiological and pathophysiological conditions have been noted $(3,4)$. In the context of bone remodeling during murine fracture repair, we demonstrated that absence of SP results in only a slight reduction of bone resorption rate but concomitantly in a critical reduction of bone formation and mineralization rate (5), whereas the absence of $\alpha$ CGRP increased the number of dysfunctional mature osteoblasts (6). Unlike bone, healthy cartilage does not contain blood vessels nor is it innervated. However, there is evidence that during $\mathrm{OA}$ progression, innervation occurs into cartilaginous tissues of the joints $(3,7)$. Suri et al. have localized SP- and $\alpha$ CGRP-positive nerve fibers in the articular cartilage of OA patients. They hypothesize that during the pathogenesis of $\mathrm{OA}$, fine unmyelinated nerves grow into joint structures through vascular channels, mainly from subchondral bone breaching through the tidemark rather than coming from synovium or periosteum (8). Of note, vascularization and innervation of the non-calcified articular cartilage zones have been found in a wide range of histological OA stages and are not restricted to endstage OA (3). Neither the mechanisms that drive innervation of cartilage in OA, nor its impact on the course of the disease have been determined. It is known that cartilage metabolism is modulated and influenced by neurotransmitters released either from nerve fibers located in neighboring tissue or directly from chondrocytes $(3,4,9)$. Chondrocytes express SP and $\alpha$ CGRP as well as the receptors for both neurotransmitters, and are responsive to peripheral neuronal stimuli $(3,10)$. Thus, it is of great interest to understand the effects of sensory nerve fibers and their neurotransmitters on joint OA pathology and how they affect chondrocytes from degraded cartilage in comparison to chondrocytes from healthy cartilage. This study aimed to analyze the influence of the sensory neuropeptides, SP and $\alpha$ CGRP, on chondrocytes from OA-patients and non-OA, healthy donors, in terms of cell vitality and metabolism, chondrogenic and inflammatory processes and the activation level of proinflammatory signaling pathways. Our data will contribute to a better understanding of potential new application routes using these neuropeptides or their inhibitors to extend and improve the therapy spectrum for OA patients.

\section{MATERIAL AND METHODS}

\section{Isolation and Culture of OA- and Non-OA Chondrocytes}

The experiments for this study were performed with human chondrocytes from knee cartilage of OA patients who had undergone endoprothetic surgery and knee cartilage harvested from cadaver donors with healthy (termed non-OA) cartilage. We used 16 OA-patients (6 male and 10 female) and 10 non-OA donors ( 6 male and 4 female) in the study. The average age of the OA patients was 62 years (+/- 9 years) and for the non-OA donors was 30 years (+/- 11 years). The use of human tissue was approved by the ethics committee at the University of Regensburg (Az: 14-101-0189, email: ethikkommission@ klinik.ukr.de). The cartilage was mechanically crushed and digested by collagenase type 2 (Worthington, Lakewood, USA) for $16 \mathrm{~h}$ at $37^{\circ} \mathrm{C}$. The chondrocytes were then seeded at a density of 12,000 cells per $\mathrm{cm}^{2}$ in T175 vials (Corning, Corning, USA) and cultivated with $25 \mathrm{ml}$ culture medium consisting of DMEMF12 Ham (Sigma-Aldrich, St. Louis, USA), 10\% FCS (SigmaAldrich, St. Louis, USA) and 1\% Pen/Strep (Sigma-Aldrich, St. Louis, USA) in an incubator (Thermo Fisher Scientific, Waltham, USA) at $37^{\circ} \mathrm{C}$ and $5 \% \mathrm{CO}_{2}$. After 5 days, the culture medium was refreshed for the first time. The adherent chondrocytes were expanded up to a confluence of $70-80 \%$ by changing medium twice a week and either frozen in liquid nitrogen for storage or directly used for further experiments either in monolayer culture (2D) or in a fibrin gel culture system (3D). All experiments with OA chondrocytes were performed at passage 2 and with non-OA chondrocytes at passage 3 .

Chondrocytes were stimulated in all experimental set ups with $10^{-8} \mathrm{M}$ and $10^{-10} \mathrm{M}$ of either SP (Sigma-Aldrich, St. Louis, USA; \#S6883) or $\alpha$ CGRP (Bachem, Bubendorf, Switzerland; \#4013281) diluted in PBS for indicated time points.

\section{Fibrin Gel Culture (3D)}

Fibrin gels are a three-dimensional (3D) culture systems in which the cells are embedded in a fibrin matrix stabilizing the chondrogenic phenotype. Cells are supplied by diffusion of the culture medium through this matrix. OA and non-OA chondrocytes were cultivated in fibrin gels for a period of 7 days. According to Leyh et al. 2x10 (6) cells each were suspended in $10 \mu \mathrm{l}$ fibrinogen (Sigma-Aldrich, St. Louis, USA) and $18 \mu \mathrm{l}$ thrombin (Baxter, Deerfield, USA) and pipetted dropwise into the middle of a well of a 24-well plate (Sarstedt, Newton, USA) (11). Cultivation was performed with daily medium change of $500 \mu \mathrm{l}$ chondrogenic medium [composition according to (12)] including the neuropeptides SP or $\alpha$ CGRP in a concentration of $10^{-8} \mathrm{M}$ and $10^{-10} \mathrm{M}$ diluted in PBS or PBS as a control for the neuropeptide effects. The fibrin gel cultures were used for the isolation of RNA and the supernatants were used for ELISAbased analysis.

\section{Protein Extraction and Western Blot Analysis}

For protein analysis, chondrocytes were cultured in 6-Well plates (20.000-30.000 cells per well) for 2-3 days and treated with SP (Sigma-Aldrich, St. Louis, USA; \#S6883), aCGRP (Bachem, Bubendorf, Switzerland; \#4013281), GR 82334 (10-6 M, Tocris

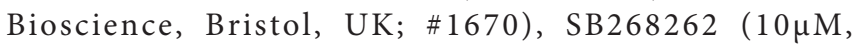
Tocris Bioscience, Bristol, UK; \#4314), U73122 (5 $\mu \mathrm{M}$, Tocris Bioscience, Bristol, UK; \#1268) or a combination thereof. Afterwards, cells were washed with PBS, detached with trypsin-EDTA (Merck, Darmstadt, Germany) and after washing again with PBS, harvested and lysed. Total cell lysates were prepared with RIPA buffer (Invitrogen/Thermo Fisher, Waltham, MA, USA) containing proteinase inhibitor (Roche, 
Munich, Germany) and phosphatase inhibitor (Roche, Munich, Germany). Protein concentration was quantified with the BCA assay (Invitrogen/Thermo Fisher, Waltham, MA, USA) and lysate aliquots containing 25-50 $\mu$ g of total protein (depending on the protein of interest) were boiled for $5 \mathrm{~min}$ with SDSsample buffer containing $\beta$-mercapto-ethanol (Merck, Darmstadt, Germany) and subjected to a $10-15 \%$ SDS-PAGE. After electrophoretic separation, the proteins were transferred to nitrocellulose membranes (Bio-Rad, Hercules, CA, USA) and blocked with 5\% dried milk (Carl Roth, Karlsruhe, Germany) and subsequently incubated with primary antibodies for $16 \mathrm{~h}$ at $4^{\circ} \mathrm{C}$ or $1 \mathrm{~h}$ at room temperature (Table 1 ):

After washing, the membranes were incubated with rabbit HRP (horseradish peroxidase)-coupled secondary antibody (1:10,000, Jackson Immuno Research, West Grove, PA, USA). Proteins were detected using ECL detection reagents (Invitrogen/ Thermo Fisher, Waltham, MA, USA). Western blot signals were analyzed densitometrically using Photoshop CS3 and the ratio between the protein of interest and $B$-actin or between the phosphorylated and not phosphorylated proteins was calculated.

\section{RNA Isolation, Reverse Transcription and Real-Time RT-PCR From Chondrocytes Cultured in 3D}

All gene expression analysis were performed with RNA isolated from fibrin gels (3D). For the isolation of RNA from fibrin gels, the MasterPure Complete RNA Purification Kit (Epicentre, Madison, USA, distributed by Biozym, Hessisch Oldendorf, Germany) was used according to Leyh et al. (11, 13). The RNA concentration was determined using a NanoDrop Spectrophotometer (260/280nm) (Thermo Fisher Scientific, Waltham, USA). To generate single-stranded cDNA, RNA was reverse transcribed with an AffinityScript QPCR cDNA Synthesis Kit (Stratagene, San Diego, CA, USA) and PCR was performed with the Mx3005P QPCR System from Agilent Technologies using Brilliant II SYBER Green qPCR Mastermix (Agilent Technologies, Santa Clara, CA, USA). Gene expression in chondrocytes were analyzed relatively according to the formula $2^{-\Delta \Delta \Delta C T}$ using the MX3005P QPCR system (Agilent Technologies, Santa Clara, USA) and were analyzed with the MxPro QPCR software for Mx3000P and Mx3005P QPCR systems (Stratagene, La Jolla, USA). The results were calibrated to the gene expression in untreated control cells, and normalized to TBP and 18s. Primer sequences are shown in Table 2.

\section{CellTiter-Blue Cell Viability Assay}

The CellTiter-Blue Cell Viability Assay (Promega, Germany) was performed to assay viability of chondrocytes stimulated with SP or $\alpha$ CGRP for 1,3 or 7 days in 2D cell culture and for 7 days in 3D cell culture (fibrin gels). 10.000 cells/well were seeded into 96well plates for 1 day of SP or $\alpha$ CGRP stimulation, 8.000 cells/well were seeded into 96-well plates for 3 days of SP or $\alpha$ CGRP stimulation and 7.000 cells/well were seeded into 48 -well plates for 7 days of SP or $\alpha$ CGRP stimulation. Chondrocytes were daily treated with SP- or $\alpha$ CGRP-containing medium. Fibrin gels were prepared with 500.000 chondrocytes embedded in 2,5 $\mu \mathrm{l}$ Fibrinogen and 4,5 $\mu$ Thrombin, and treated for 7 days with SP or $\alpha$ CGRP with daily medium changes and addition of neuropeptides. After indicated stimulation time, the amount of resazurin reduced to resorufin (pink and highly fluorescent) was determined at $579 / 584 \mathrm{~nm}$ with a Tecan ELISA reader (Maennedorf, Switzerland).

\section{Proliferation - Doubling time}

For assaying the cell growth, equal numbers of cells $(20,000$ $30,000)$ were seeded in 6-well plates in triplicates and treated with SP or $\alpha$ CGRP each day. The cells of one well were detached each day and counted with a cell counter (Cedex, Roche, Germany) for up to 6 days. The doubling time was calculated using the following formula:

Doubling Time $=($ duration $\times \log (2)) /(\log ($ Final Concentration $)-\log ($ Initial Concentration $))$

\section{Caspase-3/7 Assay}

For assaying apoptosis, equal numbers of cells (5000) were seeded in black 96-well plates in triplicates and treated with SP or $\alpha$ CGRP for 1 day. Caspase-3/7 enzymatic activity was measured as an indicator of apoptosis using the Apo-ONE Homogeneous Caspase-3/7 assay (Promega, Fitchburg, WI, USA) according to the manufacturer's instructions. A nonfluorescent caspase substrate (Z-DEVD-R110), added to the HTB94 cells, was thereby cleaved into fluorescent molecules with an emission maximum at $521 \mathrm{~nm}$ and measured in a microplate reader (Tecan, Männedorf, Switzerland).

\section{Senescence-Associated (SA)-B-Galactosidase Assay}

For assaying senescence, equal numbers of cells (5000) were seeded in 96-well plates in triplicates and treated with SP or

TABLE 1 | Antibodies used in this study.

\begin{tabular}{l} 
Antibody \\
\hline rabbit monoclonal anti-p44/42 MAPK (ERK1/2) \\
rabbit monoclonal anti-phospho-p44/42 MAPK (p-ERK1/2) \\
rabbit monoclonal anti-phospho-Akt (Ser473) (D9E) XP ${ }^{\circledR}$ \\
rabbit monoclonal anti-Akt (pan) (C67E7) \\
rabbit polyclonal anti-CRLR/CGRPR1 \\
rabbit monoclonal anti-NK1R \\
rabbit monoclonal anti-Ramp1 \\
rabbit polyclonal anti-beta actin
\end{tabular}

rabbit polyclonal anti-beta actin
Company

Dilution

Cell Signaling Technology (CST), Danvers, MA, USA; \#4695

Cell Signaling Technology (CST), Danvers, MA, USA; \#4370

Cell Signaling Technology (CST), Danvers, MA, USA; \#4060

Cell Signaling Technology (CST), Danvers, MA, USA; \#4691

Invitrogen/Thermo Fisher, Waltham, MA, USA; \#BS-1860R

Abcam, Cambridge, UK; \#ab183713

Abcam, Cambridge, UK; \#ab156575

Abcam, Cambridge, UK; \#ab8227
$1: 2000$

$1: 1000$

$1: 2000$

$1: 1000$

$1: 500$

$1: 20.000$

$1: 1000$

$1: 5000$ 
TABLE 2 | qPCR primers.

\begin{tabular}{|c|c|c|}
\hline Gene & Forward & Reverse \\
\hline $18 \mathrm{~S}$ ribosomal RNA & CTGGATACCGCAGCTAGGAA & GAATITCACCTCTAGCGGCG \\
\hline ACAN (Aggrecan) & CTATACCCCAGTGGGCACAT & GGCACTTCAGTTGCAGAAGG \\
\hline BMP4 (Bone morphogenetic protein 4) & AAGCGTAGCCCTAAGCATCA & TGGTTGAGTTGAGGTGGTCA \\
\hline COL2A1 (Collagen type II alpha 1) & CCAGATGACCTTCCTACGCC & TTCAGGGCAGTGTACGTGAAC \\
\hline COL9A1 (Collagen type IX alpha 1) & GAG CAC CGA CAG ATC AGC AC & AGT GGC ACC TGA GTC TGG A \\
\hline Cox2 (Cyclooxygenase-2) & TGC TTG TCT GGA ACA ACT GC & TGA GCA TCT ACG GTT TGC TG \\
\hline ICAM-1 (Intercellular Adhesion Molecule 1) & AGCTTCTCCTGCTCTGCAAC & GACAATCCCTCTCGTCCAGT \\
\hline IL1ß (Interleukin 1ß) & TAA GCC CAC TCT ACA GCT GG & GAG AGG TGC TGA TGT ACC AG \\
\hline IL6 (Interleukin 6) & CAA TGA GGA GAC TTG CCT GG & GCA CAG CTC TGG CTT GTT CC \\
\hline ITGA11 ((Integrin subunit alpha 11) & GCAGCAGCCTGAGCCACTAC & AGCACGACGCAAGTCTTCCTC \\
\hline P16 (Cyclin-dependent kinase inhibitor 2A, CDKN2A) & CCA ACG CAC CGA ATA GTT ACG & GCG CTG CCC ATC ATC ATG \\
\hline SIRT1 (Sirtuin-1) & GGA GCA GAT TAG TAGGCG GC & CCT CAG CGC CAT GGA AAA TG \\
\hline SOX9 (SRY-Box Transcription Factor 9) & GTACCCGCACTTGCACAAC & TCTCGCTCTCGTTCAGAAGTC \\
\hline TBP (TATA-Box Binding Protein) & GAACATCATGGATCAGAACAACA & ATAGGGATTCCGGGAGTCAT \\
\hline TNF alpha (tumor necrosis factor alpha) & CAC ATT CCT GAA TCC CAG GT & TCC TTC AGA CAC CCT CAA CC \\
\hline VEGFR (vascular endothelial growth factor) & TGAAAACTTTGGAAGACAGAACC & ACAGACTCCCTGCTाTGCT \\
\hline
\end{tabular}

$\alpha$ CGRP for 1 day. For the determination of cellular senescence, we used the Mammalian SA- $\beta$-Galactosidase Assay Kit (Thermo Fisher Scientific, Waltham, USA) according to manufacturer's instruction. Thereby, we measured the senescence-associated $\beta$ galactosidase (SA- $\beta$-gal) activity by using a fluorometric substrate, producing a bright yellow color with a peak absorbance at $420 \mathrm{~nm}$ that can be quantified using a microplate reader (Tecan, Männedorf, Switzerland).

\section{Adhesion Assay}

For measuring the adhesion of chondrocytes on cell culture dishes, 1000 cells were seeded (per well of a 96-well plate) together with SP or $\alpha$ CGRP. Culture medium and nonadhered cells were removed $30 \mathrm{~min}$ later. Cells were washed with PBS and fixed with 1\% Glutaraldehyd (Merck, Darmstadt, Germany) for $30 \mathrm{~min}$ at rt. Crystal violet solution (in 0,02\% aqua dest.) (Carl Roth, Karlsruhe, Germany) was added to the wells at rt for $15 \mathrm{~min}$. After removing of the staining solution, wells were washed with deionized $\mathrm{H}_{2} \mathrm{O}$ and crystal violet was extracted from the cells by adding $70 \%$ ethanol for $3 \mathrm{~h}$. Plates were shaken gently at room temperature for $15 \mathrm{~min}$ and $100 \mu \mathrm{l}$ of the extracted crystal violet were transferred to a flat-bottom 96-well plates. Absorbance was measured at $590 \mathrm{~nm}$ in a microplate reader (Tecan, Männedorf, Switzerland).

\section{IL6 and IL1ß-ELISAs}

The amount of IL6 or IL1ß in the cell culture supernatant of the fibrin gel cultures was measured according to manufacturer's instruction using a human IL-6 ELISA (Ray Biotech, Norcross, GA, US) or a human IL-1ß ELISA (R\&D, Minneapolis, MN, US). Chondrocytes in fibrin gels were daily treated with SP or $\alpha$ CGRP for 7 days and supernatant samples were harvested on the last day. Standards and samples are pipetted into the wells of an antibody pre-coated plate and IL-6 or IL1ß present in a sample or standard is bound by the immobilized antibody. The standard curve is used to determine the concentration of the samples. Standards and samples are measured at $450 \mathrm{~nm}$ in a microplate reader (Tecan, Männedorf, Switzerland).

\section{Collagen Type II ELISA and DMMB Assay}

Chondrocytes were cultured in fibrin gels and treated daily with SP or $\alpha$ CGRP for 7 days. Fibrin gels were homogenized and digested with pepsin $(10 \mathrm{mg} / \mathrm{ml}$ in $0.05 \mathrm{M}$ acetic acid containing 0.4 M NaCl, Sigma-Aldrich, St. Louis, MO, USA) for $48 \mathrm{~h}$ at $4^{\circ} \mathrm{C}$ and further digested with elastase $(1 \mathrm{mg} / \mathrm{ml}$ in Tris-buffered saline (TBS) pH 8; Serva Electrophoresis, Heidelberg, Germany) for $24 \mathrm{~h}$ at $4^{\circ} \mathrm{C}$. Samples were stored at $-20^{\circ} \mathrm{C}$ until they were analyzed for GAG (glycosaminoglycan) or collagen type II content by ELISA.

Collagen type II content of digested fibrin gels were measured with specific sandwich ELISA, which recognize the native conformation of collagen type II chains (Chondrex, Redmond, WA, USA), according to the manufacturer's instructions and measured in an ELISA-reader (Tecan, Männedorf, Switzerland) at $490 \mathrm{~nm}$. All measurements were performed in triplets.

GAG content of digested fibrin gels were measured by DMMB (dimethyl methylene blue) (Merck, Darmstadt, Germany) assay. DMMB specifically stains chondroitin sulphate. Aliquots $(25 \mu \mathrm{l})$ of the sample or of a standard dilution series (1:2) of known concentrations of chondroitin sulphate A (Merck, Darmstadt, Germany), used for calibration, were mixed with $250 \mu \mathrm{l} \mathrm{DMMB}$ staining solution ( $2 \mathrm{ml}$ formic acid and $2 \mathrm{~g}$ sodium formate were dissolved in distilled water, $\mathrm{pH}$ 3.0 adjusted with $\mathrm{HCl} ; 18 \mathrm{mg}$ DMMB solved in $5 \mathrm{~mL}$ ethanol and added) and measured in an ELISA-reader (Tecan, Männedorf, Switzerland) at $595 \mathrm{~nm}$. All measurements were performed in triplicate.

\section{Gelatin Zymography}

Gelatin zymography was performed to analyze MMP-2 or -9 activity with chondrocytes cultured either in $2 \mathrm{D}$ monolayer or $3 \mathrm{D}$ fibrin gels. For monolayer culture 50.000 cells/well were seeded into 6-well plates. Chondrocytes were cultured for 3 days with daily addition of fresh medium containing SP or $\alpha$ CGRP. For the last 24 hours, cells were cultured in serum-free medium containing SP or $\alpha$ CGRP. Fibrin gels were prepared with 500.000 chondrocytes embedded in 2,5 $\mu \mathrm{l}$ Fibrinogen and $4,5 \mu \mathrm{l}$ 
Thrombin, and treated for 7 days with SP or $\alpha$ CGRP with daily medium changes and addition of neuropeptides Equal amount of supernatants were mixed with $5 x$ loading buffer (20\% glycerol, 2\% SDS, 2mM EDTA, 0.02\% bromophenol blue, $20 \mathrm{mM}$ Tris/ $\mathrm{HCl}, \mathrm{pH} 8.0$ ) and subjected to electrophoresis (10\% SDSpolyacrylamide gels supplemented with $1 \%$ gelatin). Subsequently, gels were washed twice for $30 \mathrm{~min}$ in Triton-X $100(2,5 \%)$ (Sigma-Aldrich, US) and in distilled water, and were developed for 1 day at $37^{\circ} \mathrm{C}$ in Tris/ $\mathrm{HCl}(50 \mathrm{mM}), \mathrm{pH} 8.5$, containing $\mathrm{CaCl} 2(5 \mathrm{mM})$. Finally, the gels were stained with Coomassie Brilliant Blue R250 (Serva, Germany) to detect protease activity. Signals were analyzed densitometrically using Photoshop CS3.

\section{MMP13 Western Blot}

50.000 cells/well were seeded into 6-well plates. Chondrocytes were cultured for 3 days with daily addition of fresh medium containing SP or $\alpha$ CGRP. For the last 24 hours, cells were cultured in serum-free medium containing SP or $\alpha$ CGRP with or without addition of IL1ß $(1 \mathrm{ng} / \mu \mathrm{l})$ for $24 \mathrm{~h}$ (Thermo Fisher Scientific, Waltham, USA) known as an inducer of proteases. Fibrin gels were prepared with 500.000 chondrocytes embedded in 2,5 $\mu \mathrm{l}$ Fibrinogen and 4,5 $\mu \mathrm{l}$ Thrombin, and treated for 7 days with SP or $\alpha$ CGRP and with or without addition of IL1ß $(1 \mathrm{ng} / \mu \mathrm{l})$ for $24 \mathrm{~h}$ with daily medium changes and addition of neuropeptides. Equal amount of supernatants were mixed with $5 x$ loading buffer (20\% glycerol, $2 \%$ SDS, 2 mM EDTA, $0.02 \%$ bromophenol blue, $20 \mathrm{mM}$ Tris/ $\mathrm{HCl}, \mathrm{pH} 8.0$ ) and subjected to electrophoresis. MM13 was detected using anti-rabbit polyclonal antibody to MMP13 (1:6000, 1 hour RT in 5\% dry milk, Abcam, \#ab39012) and rabbit horseradish peroxidise-coupled secondary antibody; 1:10.000, Jackson Immuno Research, US). Protein bands were detected with ECL detection reagents (Thermo Fisher Scientific, US).

\section{CAMP ELISA}

For assaying the intracellular cyclic AMP (cAMP) level equal numbers of cells $(20,000-30,000)$ were seeded in 6-well plates in triplicate. After preincubation with Rolipram (Sigma-Aldrich, St. Louis, USA) and following treatment with forskolin (SigmaAldrich, St. Louis, USA), SP or $\alpha$ CGRP, cAMP was measured using the cAMP ELISA Kit (Colorimetric) (Cell Biolabs Inc., San Diego, CA, USA) according to the manufacturer's instruction. The cAMP-ELISA is a competitive enzyme immunoassay, using an anti-Rabbit IgG polyclonal coating antibody being adsorbed onto a microtiter plate. Cyclic AMP present in the sample or standard competes with Peroxidase cAMP Tracer for plate binding, in the presence of Rabbit Anti-cAMP Polyclonal Antibody. Following incubation and wash steps, a Peroxidase cAMP Tracer bound to the plate is detected with addition of substrate solution. The colored product formed is inversely proportional to the amount of cAMP present in the sample. The reaction is terminated by addition of a Stop solution and absorbance is measured at $450 \mathrm{~nm}$. A standard curve is prepared from cAMP standard and sample concentration is then determined.

\section{Statistics}

Statistical analysis was performed using Prism 6 (GraphPad Software Inc., San Diego, CA, USA). Results are presented in boxplots (median, min to max) or columns (mean, SD). Each assay was performed in replicates and repeated at least in three independent experiments. One sample t-tests were used to determine if the mean of the different groups (experimental group vs. control) were significantly different. Exact P-values were calculated. A value of $\mathrm{p} \leq 0.05$ was considered statistically significant. For the semi-quantitative analysis of Western blot intensity (or if specifically denoted), unpaired t-tests were used.

\section{RESULTS}

\section{Production of SP and $\alpha$ CGRP and Their Receptors NK1-R and CLRL/Ramp1 in OA- and Healthy Chondrocytes}

We analyzed the concentration of SP and $\alpha$ CGRP in the culture supernatants of chondrocytes from OA patients $(\mathrm{OA}-\mathrm{CH})$ and healthy donors (non-OA-CH). Whereas for SP no differences were found between both groups (OA-CH: 22,91 $\pm 4,762 \mathrm{pg} / \mathrm{ml}$ vs. non-OA-CH: $24,85 \pm 3,218 \mathrm{pg} / \mathrm{ml}$ ) (Figure 1A), the level of $\alpha C G R P$ was increased by trend in the supernatant of $\mathrm{OA}-\mathrm{CH}$ $(191,3 \pm 93,77 \mathrm{pg} / \mathrm{ml})$ compared to non-OA-CH $(88,79 \pm 36,00$ $\mathrm{pg} / \mathrm{ml}$ ), although not statistically significantly (Figure 1B). The protein expression of neurokinin-1 receptor (NK1R), the receptor with the highest affinity for SP, was similar for OAand non-OA-CH (Figures 1C, D). The receptor for $\alpha C G R P$ (CGRP-R) consists of two transmembrane protein subunits, the calcitonin receptor-like receptor (CRLR) unit and the receptor activity-modifying protein 1 (RAMP1) unit, with the latter subunit providing the specificity for $\alpha$ CGRP. The signal of the band corresponding to RAMP1 was significantly weaker in OA$\mathrm{CH}$, whereas the band signal intensity of CRLR in OA-CH was similar to that in non-OA-CH (Figures 1C, D).

$\mathrm{OA}$ and non-OA chondrocytes produced and secreted SP and $\alpha C G R P$ in comparably low concentrations (pg/ml). Non-OA chondrocytes showed higher expression of the $\alpha$ CGRP receptor subunit RAMP-1 compared to OA chondrocytes.

\section{Effects of SP and $\alpha$ CGRP on Viability, Proliferation, Apoptosis, Senescence and Adhesion}

First, we analyzed effects of SP and $\alpha$ CGRP treatment on viability of OA- and non-OA chondrocytes at different culture time points. Both concentrations of SP reduced viability of $\mathrm{OA}-\mathrm{CH}$ at the first day of culture but not at later culture time points compared to untreated controls. Also, both concentrations of $\alpha$ CGRP reduced viability of non-OA-CH at day 7 - the end point- of culture compared to untreated controls (Figures 2A-C). Viability of chondrocytes kept in 3D fibrin gel culture was not affected at any time point (Figure 2D).

We compared the proliferation rate of OA- and non-OA-CH to their respective untreated control cells by counting the cell 
A

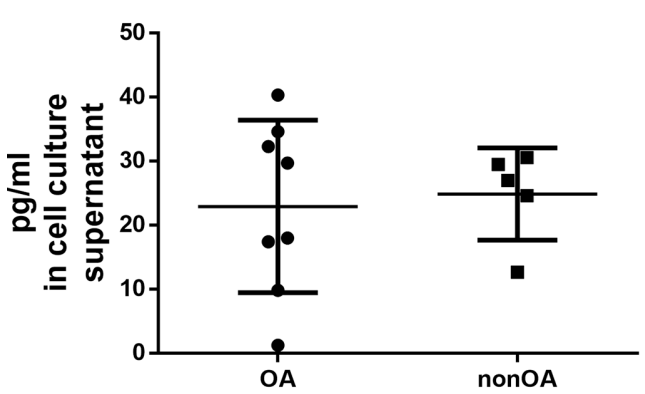

C

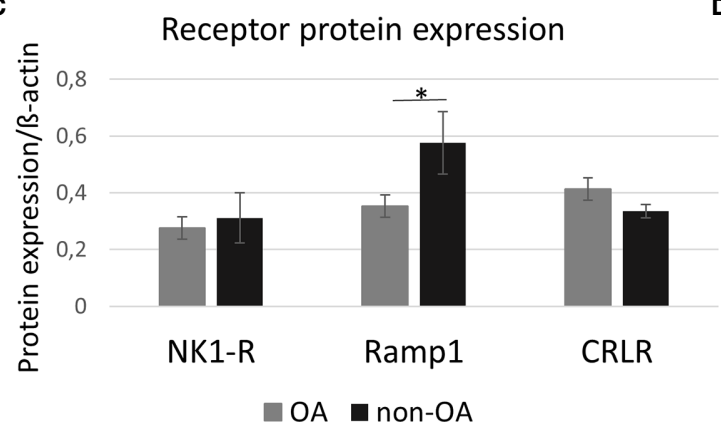

B
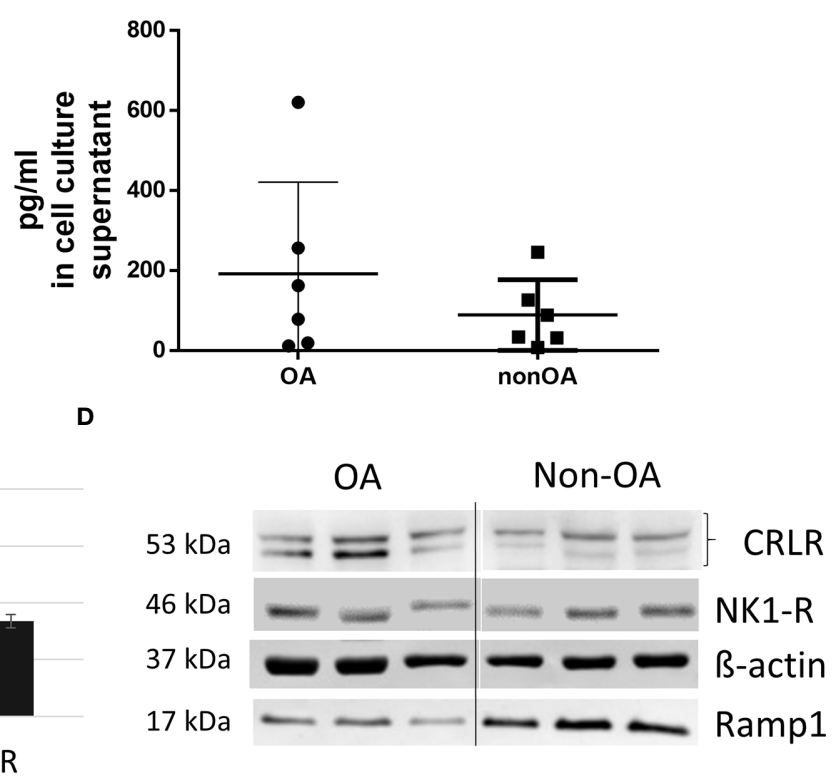

FIGURE 1 | Protein biosynthesis of SP, $\alpha$ CGRP, NK1-R and CGRP-R (CRLR and Ramp1) in OA and non-OA chondrocytes. (A, B) Protein concentration of SP and $\alpha C G R P$ was determined in the cell culture supernatants with ELISA, $n=5-8$. (C) Relative receptor protein expression was determined densitometrically. Expression of $\beta$-actin served as endogenous loading control, $n=3$. (D) Representative Western Blot images showing CRLR ( 53 kDa), NK1-R ( 46 kDa), Ramp1 ( 17 kDa) and $\beta$-actin $(\sim 37 \mathrm{kDa}$ ) protein bands in chondrocyte lysates from OA- and non-OA donors ( $n=3$ different donors for each group). Results are means $+/-\mathrm{SD}$; one sample t-test * $\mathrm{p} \leq$ 0,05; OA, osteoarthritis; SP, substance P; $\alpha \mathrm{CGRP}$, alpha-calcitonin gene-related peptide; NK1-R, neurokinin 1 receptor; CRLR, calcitonin receptor-like receptor; Ramp1, receptor activity modifying protein 1.

numbers after 3 and 7 days of stimulation with SP or $\alpha$ CGRP in two different concentrations $\left(10^{-8} \mathrm{M}\right.$ or $\left.10^{-10} \mathrm{M}\right)$. After 3 days of stimulation with $10^{-8} \mathrm{M}$ SP, OA-CH had proliferated less compared with control cells (Figure 3A). This effect was lost after 7 days, but by then, stimulation with $10^{-8} \mathrm{M} \alpha \mathrm{CGRP}$ leads to a significantly reduced proliferation rate in $\mathrm{OA}$ - and non-OA$\mathrm{CH}$ (Figure 3B).

Apoptosis was also influenced by the two neuropeptides. Stimulation with SP $\left(10^{-8} \mathrm{M}\right)$, and also with $\alpha$ CGRP $\left(10^{-8} \mathrm{M}\right.$ and $10^{-10} \mathrm{M}$ ) for 1 day, increased caspase $3 / 7$ activity in OA$\mathrm{CH}$, indicating enhanced apoptosis in those cells. In contrast, $10^{-10} \mathrm{M} \alpha \mathrm{CGRP}$ has beneficial effects on non-OA-CH by decreasing apoptosis significantly (Figure 3C).

As apoptosis is often closely related to cellular senescence, we measured the senescence-associated (SA)- $B$-galactosidase activity as an indicator for senescence-related processes in OAand non-OA-CH after 1 day of addition of SP and $\alpha$ CGRP. OA$\mathrm{CH}$ respond with an enhanced $\mathrm{SA}-\mathrm{B}$-galactosidase activity to addition of $10^{-8} \mathrm{M}$ SP and $\alpha$ CGRP (Figure 3D). Even though there is no universal marker for cell senescence, most senescent cells increase expression of p16 (p16 ink4A $)$, a cell cycle inhibitor that targets cyclin-dependent kinases (CDKs). Thus, we analyzed the gene expression of p16 after 7 days of incubation with both neuropeptides. $\alpha$ CGRP exerted opposite effects on OA- and non-
$\mathrm{OA}-\mathrm{CH}$. Whereas in non-OA-CH, $\alpha \mathrm{CGRP}$ reduced the expression of $\mathrm{p} 16$, in $\mathrm{OA}-\mathrm{CH} \alpha \mathrm{CGRP}$ induced gene expression of this senescence marker (Figure 3E) in comparison with untreated control cells.

Chondrocyte adhesion is critical in cell based regenerative strategies for treatment of focal cartilage defects. As SP and $\alpha$ CGRP might be of interest for therapeutic approaches, we investigated the adhesion behavior of OA- and non-OA-CH during stimulation with the two neuropeptides. Both neuropeptides decreased the number of adherent non-OA-CH at 30 minutes after seeding; SP in a low concentration $\left(10^{-10} \mathrm{M}\right)$ and $\alpha$ CGRP in a high concentration $\left(10^{-8} \mathrm{M}\right)($ Figure $3 \mathrm{~F})$. As we have observed direct effects of SP and $\alpha$ CGRP on different cellular metabolic properties like viability, proliferation, apoptosis, senescence and adhesion in OA- and non-OA-CH, we analyzed the metabolic activity of SP- or $\alpha$ CGRP-treated cells using a real-time metabolic bioanalyzer (Seahorse XF). No significant differences between any groups were detected (Supplementary Figure S1).

Both neuropeptides affected metabolism of OA- and non-OA chondrocytes differently. SP stimulation mostly affected OAchondrocytes whereas in non-OA chondrocytes, SP only modulated adhesion capability. Contrary, both OA- and nonOA chondrocytes responded to $\alpha$ CGRP stimulation. 


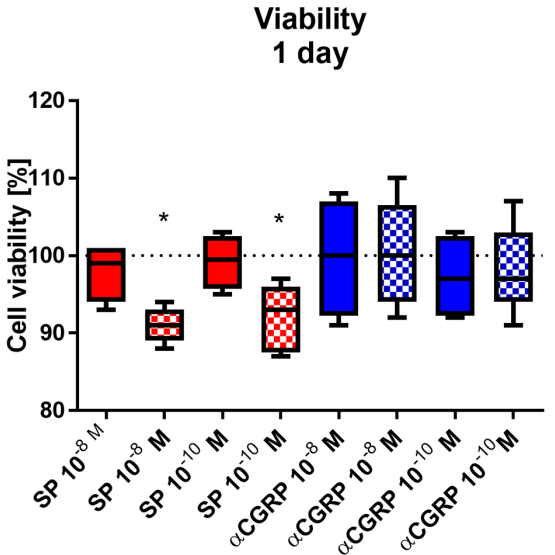

C

Viability
7 days

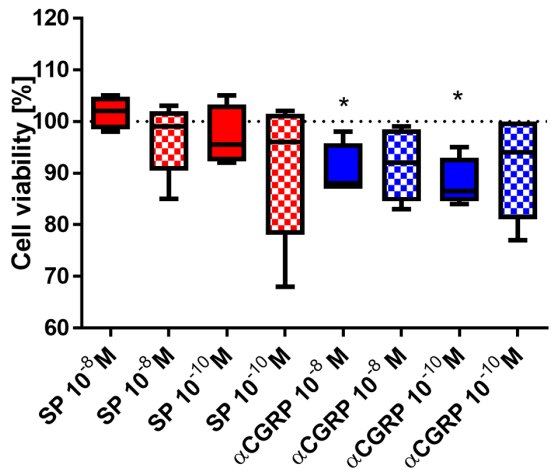

B
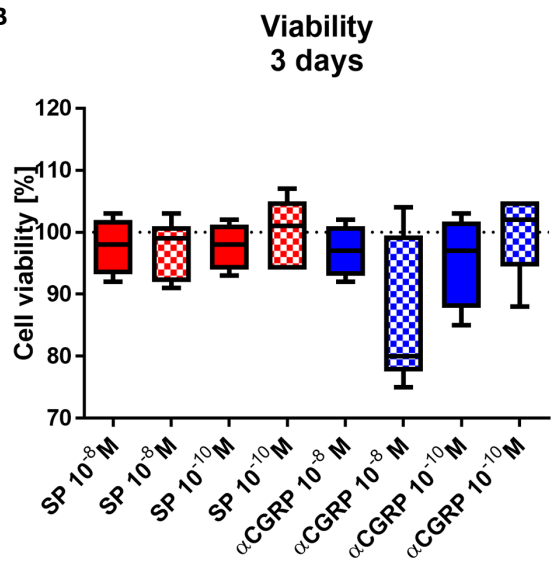

D

Viability

7 days - (3D culture)

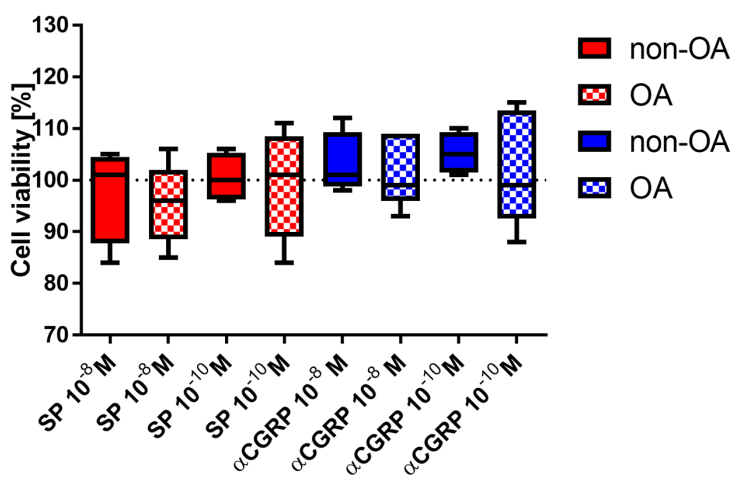

FIGURE 2 | Effects of SP and $\alpha$ CGRP stimulation on viability of OA and non-OA chondrocytes. Chondrocytes were treated with either $10^{-8}$ or $10^{-10} \mathrm{M} \mathrm{SP}$ or $\alpha \mathrm{CGRP}$ and then compared with the respective untreated control cells (untreated controls set to $100 \%=$ dotted line). Viability was determined in monolayer culture after 1 day (A), 3 days (B) and 7 days (C). (D) Viability was determined in 3D fibrin gel culture after 7 days. Results show median (min to max); one sample t-test; ${ }^{*} \mathrm{p} \leq 0,05$; $n=5$.

\section{Expression of Chondrogenic and Mesenchymal Markers in OA- and Healthy Chondrocytes After SP and $\alpha$ CGRP Stimulation}

To detect the influence of SP and $\alpha$ CGRP on chondrogenic/ mesenchymal marker genes, we analyzed the expression levels of SOX9, ACAN, BMP4, VEGFR, ITGA11, COL9A1 and COL2A1 (Figures 4A-G). The OA- and non-OA-CH were encapsulated in fibrin gels for 7 days with daily SP or $\alpha$ CGRP addition and the gene expression levels were compared with the respective untreated control chondrocytes. We measured decreased gene expression of SOX9, ACAN (Figures 4A, B) and ITGA11 (Figure 4E) in OA$\mathrm{CH}$ after addition of $10^{-8}$ and $10^{-10} \mathrm{M}$ SP, and of COL9A1 and COL2A1 in the presence of $10^{-10} \mathrm{M} \mathrm{SP}$ (Figures 4F, G). In contrast, the gene expression levels of BMP-4 (Figure 4C), VEGFR (Figure 4D), ITGA11 (Figure 4E) and COL9A1 (Figure 4F) were increased in non-OA-CH after $10^{-8} \mathrm{M} \alpha \mathrm{CGRP}$ addition. In contrast to the effects on non-OA-CH, the gene expression of COL2A1 (Figure 4G), COL9A1 (Figure 4F) and ITGA11 (Figure 4E) gene expression were lower in $\mathrm{OA}-\mathrm{CH}$ after $10^{-8} \mathrm{M}$ $\alpha \mathrm{CGRP}$ addition. COL9A1 gene expression in $\mathrm{OA}-\mathrm{CH}$ was also decreased by $10^{-10} \mathrm{M} \alpha \mathrm{CGRP}$ stimulation (Figure 4F). The biosynthesis of collagen type II was not affected (Supplementary Figure S2) in either chondrocyte group after SP or $\alpha$ CGRP addition, but a decreased glycosaminoglycan (GAG) content in $\mathrm{OA}-\mathrm{CH}$ containing fibrin gels after incubation with $10^{-10} \mathrm{M}$ $\alpha$ CGRP was observed (Figure 4H).

Stimulation with SP affected only gene expression in OAchondrocytes (reduction of anabolic gene expression of SOX9, ACAN, ITGA11, COL9A1, COL2A1) but not in non-OA chondrocytes. Stimulation with $\alpha$ GRRP induced gene expression (BMP4, VEGFR, ITGA11, COL9A1) in non-OA chondrocytes but reduced gene expression (ITGA11, COL9A1, COL2A1) in OA-chondrocytes. However, protein synthesis of collagen II remained unaffected as did mostly GAG synthesis except in OA chondrocytes after $\alpha$ CGRP stimulation.

\section{Expression of Inflammatory Markers in OA- and Healthy Chondrocytes After SP and $\alpha$ CGRP Stimulation}

SP and $\alpha C G R P$ effects are often related to inflammatory processes in various tissues and can influence the expression of 

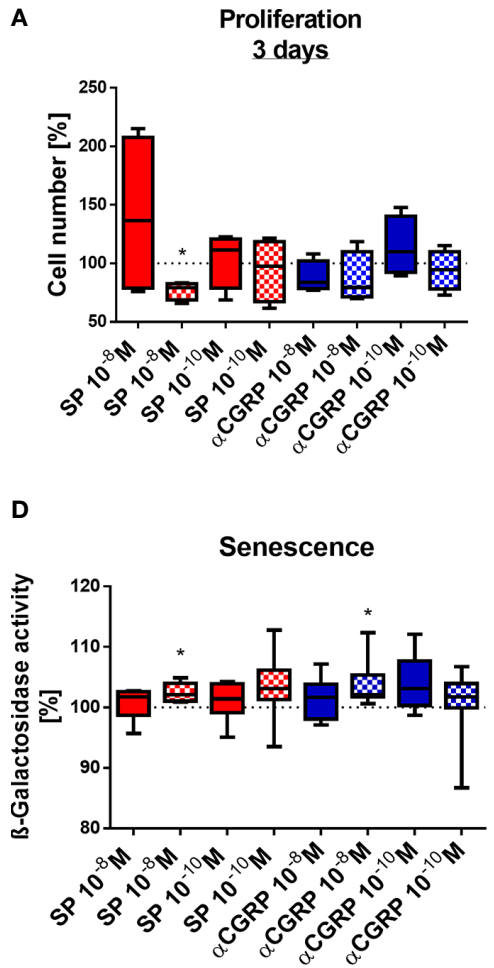

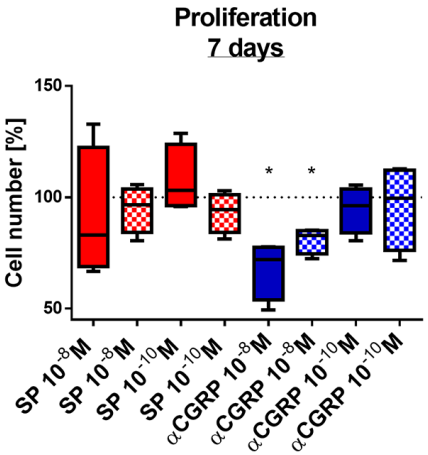

E

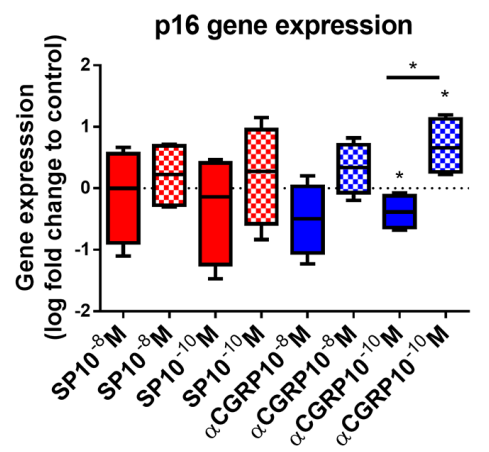

C Apoptosis

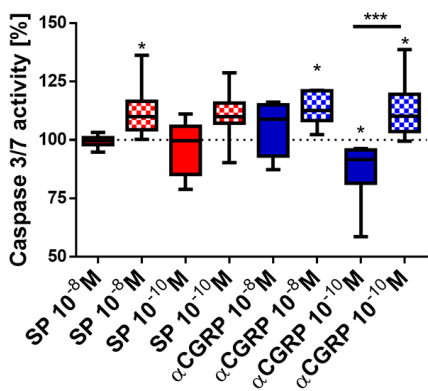

$\mathbf{F}$

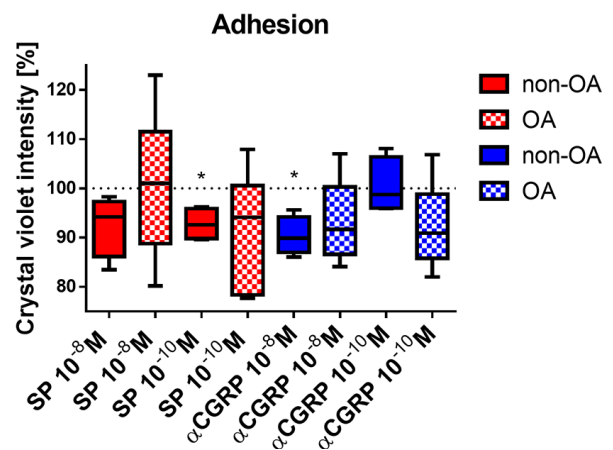

FIGURE 3 | Effects of SP and $\alpha$ CGRP stimulation on proliferation, apoptosis, senescence and adhesion of OA and non-OA chondrocytes. Chondrocytes were treated with either $10^{-8}$ or $10^{-10} \mathrm{M} \mathrm{SP}$ or $\alpha$ CGRP and then compared with the respective untreated control cells (untreated controls set to $100 \%=$ dotted line). (A, B) Proliferation was determined after 3 days and 7 days of SP/ $\alpha$ CGRP stimulation by cell counting. (C) Apoptosis was measured with a caspase $3 / 7$ assay after 1 day of SP/ $\alpha$ CGRP stimulation. (D, E) Analysis of SA-B-galactosidase activity and p16 gene expression revealed the senescence status of the cells after 1 day of SP/ $\alpha$ CGRP stimulation. (F) The adhesion capacity was analysed via crystal violet staining after 30 minutes of SP and $\alpha C G R P$ stimulation. Results show median (min to max); one sample t-test; ${ }^{*} p \leq 0,05 ;{ }^{* \star *} p \leq 0,001 ; n=4-7$.

pro-inflammatory cytokines. We therefore analyzed gene expression of TNF $\alpha$, IL6, IL1ß, ICAM and COX2 (Figures 5AE) and protein concentration of IL6 and IL1ß in culture supernatants (Figures 5F, G) after stimulation with SP or $\alpha C G R P$. In $\mathrm{OA}-\mathrm{CH}, 10^{-8} \mathrm{M}$ SP resulted in an increased gene expression of TNFo, IL6 and IL1ß (Figures 5A-C). The gene expression of IL1ß was also increased when $\mathrm{OA}-\mathrm{CH}$ were subjected to a lower dose of SP $\left(10^{-10} \mathrm{M}\right)$ (Figure 5C). The gene expression of the transmembrane protein ICAM-1 (CD54), a cell surface glycoprotein known for regulating leukocyte recruitment from circulation to sites of inflammation, was reduced in $\mathrm{OA}-\mathrm{CH}$ after treatment with $10^{-8} \mathrm{M}$ SP (Figure 5D). In non-OA-CH, $10^{-8} \mathrm{M}$ and $10^{-10} \mathrm{M}$ SP increased the gene expression of COX2 (Figure 5E), a key mediator of pro-inflammatory pathways, and $10^{-8} \mathrm{M}$ SP increased the gene expression level of IL6 in non-OA$\mathrm{CH}$ compared to control cells (Figure 5B). We also measured protein levels in cells with altered IL6 or IL1ß gene expression. IL6 protein amount was increased in the supernatant of non-OA-CH that had been exposed to $10^{-8} \mathrm{M} \mathrm{SP}$, in comparison with controls (Figure 5F). However, in the OA-CH group, neither the IL6 (Figure 5F) nor IL1ß (Figure 5G) concentrations in the supernatants were affected by $10^{-8} \mathrm{M}$ SP treatment. Notably, $\alpha$ CGRP did not affect any of the analyzed inflammatory markers neither in OA- nor in non-OA-CH (Supplementary Figures S3A-E). Notably, the IL1ß concentration in non-OA-CH supernatants was below the ELISA detection limit. In addition, the expression and activity of different MMPs was analyzed in OA- CH after SP stimulation. MMP-9 was not detectable at all and MMP-13 was mostly only detectable after IL1ß stimulation in $3 \mathrm{D}$ culture only and not affected by SP stimulation. Activity of pro- and active forms of MMP-2 was not affected by SP stimulation neither in 2D- nor in 3D culture (Supplementary Figures S4A-E).

Expression of pro-inflammatory genes remained unaffected by $\alpha$ CGRP stimulation but was induced by SP stimulation (except ICAM gene expression which was reduced) in both chondrocyte groups. In addition, SP stimulation increased IL6 concentration in culture supernatant of non-OA CH.

\section{Activation of cAMP-Dependent Pathways in OA- and Healthy Chondrocytes}

G-protein-coupled receptors (GPCR) like NK1-R and CLRL/ Ramp1 (CGRP-R) are able to activate different signaling pathways via different alpha-subunits. To elucidate the 
A

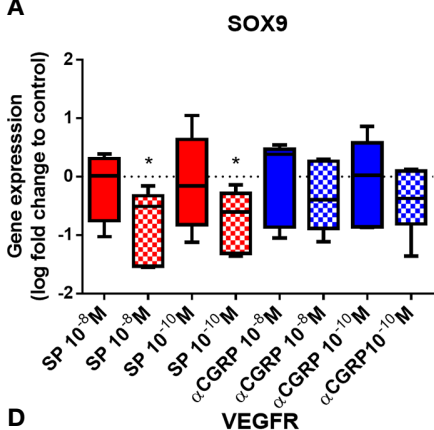

D

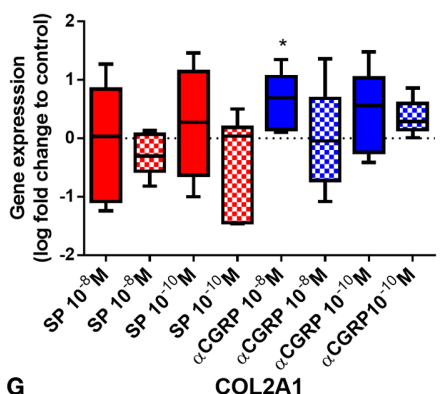

G

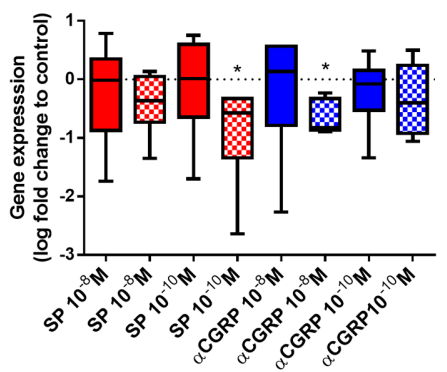

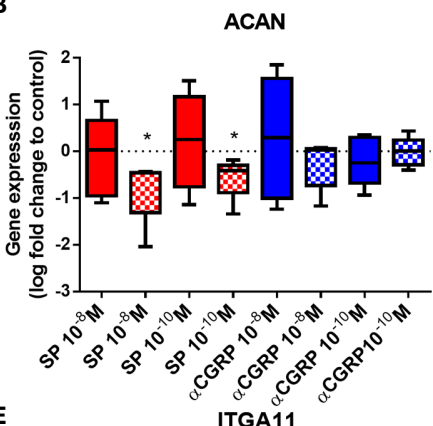

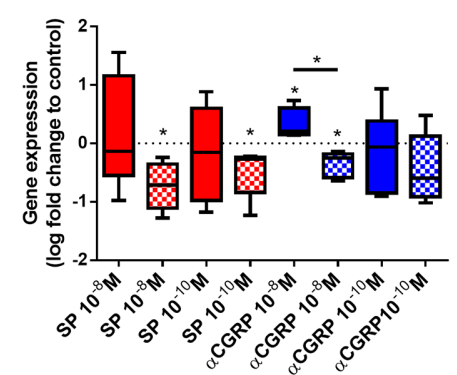

H

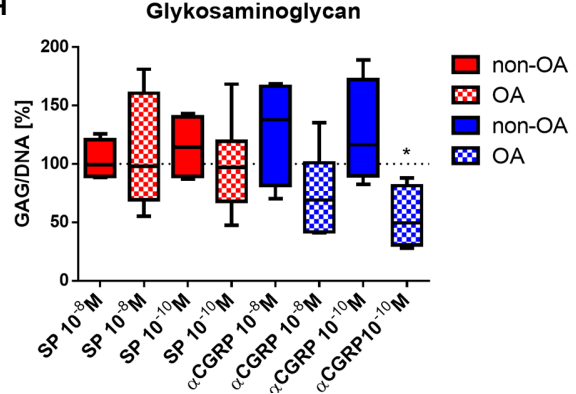

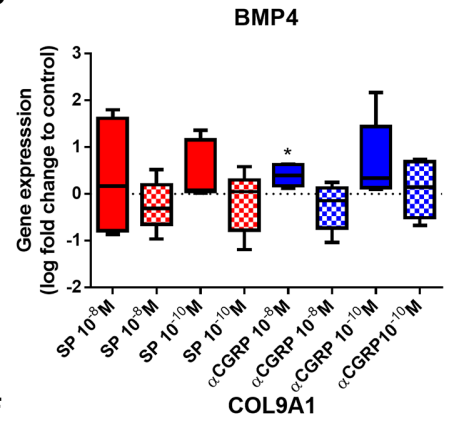

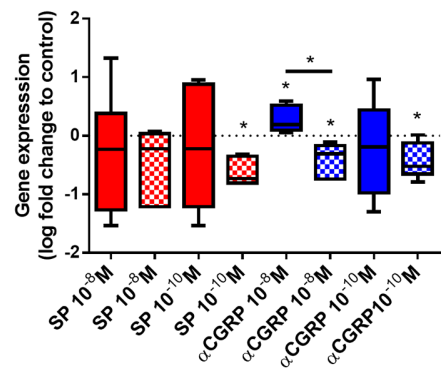

FIGURE 4 | Expression of chondrogenic and mesenchymal markers in 3D fibrin gels cultured OA- and non-OA chondrocytes after stimulation with SP and $\alpha C G R P$. Chondrocytes were treated with either $10^{-8}$ or $10^{-10} \mathrm{M} \mathrm{SP}$ or $\alpha$ CGRP for 7 days and then compared to the respective untreated control cells (untreated controls set to 0 or $100 \%$ = dotted line). (A-G) Quantitative RT-PCR analysis revealed the gene expression of SOX9, ACAN, BMP4, VEGFR, ITGA11, COL9A1 and COL2A1. (H) The glycosaminoglycan (GAG) content in OA and non-OA chondrocytes was analysed with a DMMB assay. Results show median (min to max); one sample t-test; ${ }^{*} \mathrm{p} \leq 0,05 ; \mathrm{n}=5-6$.

signaling mechanism in $\mathrm{OA}$ - and non-OA-CH after SP and $\alpha C G R P$ treatment, we examined the level of the second messenger cyclic adenosine-mono-phosphate (cAMP), which is known to be activated after assembling of GPCRs with Gos subunits. To do this, we pre-incubated the cells with Rolipram, a selective inhibitor of type 4 cyclic nucleotide phosphodiesterases (PDE4), which mediates cAMP degradation. In non-OA-CH a high concentration $\left(10^{-8} \mathrm{M}\right)$ of $\mathrm{SP}$ and $\alpha$ CGRP provoked a comparable increase in the cAMP-level (Figure 6A). In contrast, in $\mathrm{OA}-\mathrm{CH}$, SP did not induce alterations in the cAMP level, but $\alpha$ CGRP increased the amount of cAMP significantly when applied in a concentration of $10^{-10} \mathrm{M}$, and by trend also at $10^{-8} \mathrm{M}$ (Figure 7A). It is noteworthy that in non$\mathrm{OA}-\mathrm{CH}$ a markedly stronger cAMP response was generated by forskolin treatment, which represents the internal positive treatment control (non-OA-CH: cAMP mean after forskolin:
2658 pM (+/-1574); OA-CH: cAMP mean after forskolin: 699 pM (+/-183)).

$\alpha C G R P$ stimulation provoked increased cAMP concentrations in non-OA and OA, whereas SP only induced cAMP level in non$\mathrm{OA}-\mathrm{CH}$.

\section{PLC-Dependent Activation of ERK and AKT Pathways in OA- and Healthy Chondrocytes}

The activation of the ERK and AKT signaling pathways is a critical step in the regulation of chondrocyte gene expression in a chronic inflammatory state. Here, we analyzed if SP or $\alpha$ CGRP can induce the phosphorylation of ERK or AKT in OA- and non$\mathrm{OA}-\mathrm{CH}$. In general, non-OA-CH developed a stronger response to the treatment with SP and $\alpha \mathrm{CGRP}$ than OA-CH (Figures 6 and 7). Both neuropeptides induced phosphorylation of ERK 

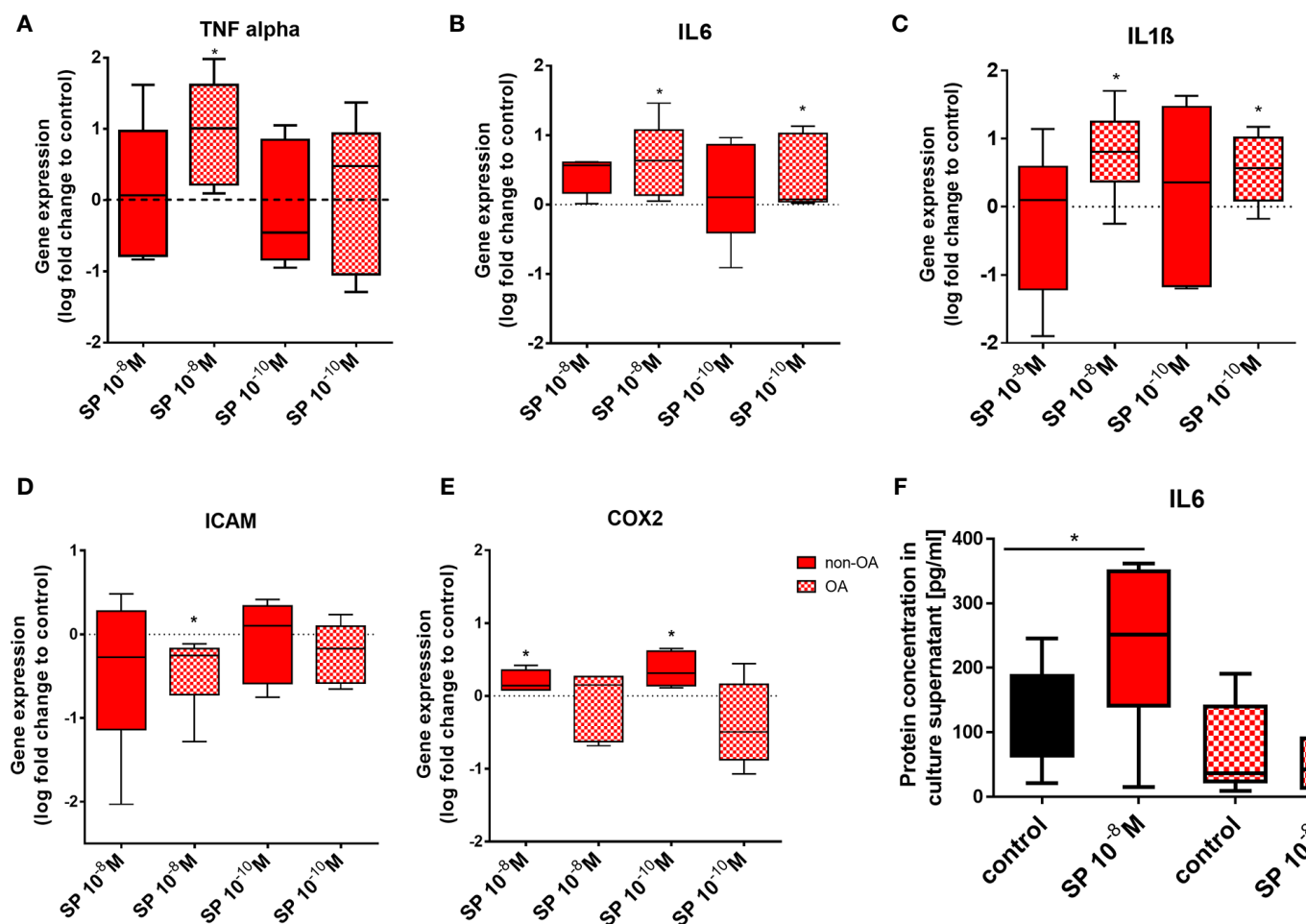

$\mathbf{F}$

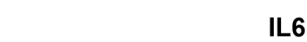

G

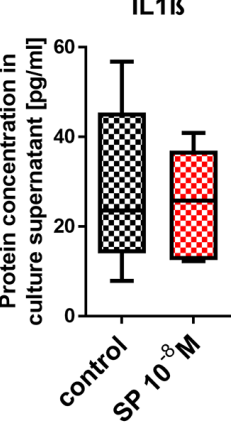

FIGURE 5 | Pro-inflammatory markers in 3D fibrin gels cultured OA and non-OA chondrocytes after stimulation with SP. Chondrocytes were treated with either $10^{-8}$ or $10^{-10} \mathrm{M} \mathrm{SP}$ for 7 days and then compared to the respective untreated control cells (untreated controls set to 0 or $100 \%=$ dotted line). (A-E) Quantitative RT-PCR analysis demonstrated the gene expression of TNF $\alpha$, IL6, IL1B, ICAM and COX2. (F, G) Protein concentration of IL6 and IL1B in the culture supernatants was assayed via ELISA. Results show median (min to max); one sample t-test; ${ }^{*} \mathrm{p} \leq 0,05 ; \mathrm{n}=5-6$.

and AKT within 5-15 minutes without alteration of the unphosphorylated ERK and AKT protein concentration in non-OA-CH (Figures 6B-D).

We hypothesized that the receptors for SP and $\alpha$ CGRP (NK1$\mathrm{R}$ and CGRP-R) can assemble not only with Gos but also with the $\alpha q$ subunit $(\mathrm{Gq})$ in chondrocytes, as this is often the case when ERK and AKT pathways are activated. Alpha q proteins couple to G proteins of GPCR to activate beta-type phospholipase C (PLC- $\beta$ ) enzymes which modulate the formation of phosphoinositol -3-phosphate (PIP3) which in turn is responsible for anchoring $\mathrm{AKT} / \mathrm{PKB}$ at the membrane and thus modulates signaling. We therefore used a specific PLC$ß$ inhibitor (U73122) in combination with SP and $\alpha$ CGRP to test this hypothesis. The phosphorylation of ERK and AKT can be blocked when non-OA-CH are treated with SP and $\alpha$ CGRP together with U73122 (Figures 6E-G), indicating an involvement of PLC- $\beta$ in this signaling pathway. In OA-CH, the induction of ERK phosphorylation via SP and $\alpha$ CGRP treatment was detected within 5 minutes (Figure 7B), but the ratio of phospho-ERK to total ERK was lower compared with that in non-OA-CH in the presence of SP (Figure 7C). The phosphorylation of ERK via $\alpha C G R P$ in OA-CH was comparable with that seen in non-OA$\mathrm{CH}$ (Figure 7C). In contrast, the AKT pathway could not be activated via SP or $\alpha$ CGRP treatment in $\mathrm{OA}-\mathrm{CH}$ (Figures $7 \mathbf{B}, \mathbf{D}$ ). Treatment of OA-CH with the specific PLC- $ß$ inhibitor U73122 in combination with SP and $\alpha$ CGRP lead to an inhibition of ERK phosphorylation (Figures 7E, F), similar to the effect seen in non$\mathrm{OA}-\mathrm{CH}$. The unchanged levels of phospho-AKT in control, SPand $\alpha C G R P$-treated OA-CH decreased slightly after PLC- $\beta$ inhibition (Figures 7E, G).

To further show that the induction of ERK and AKT phosphorylation is SP- and $\alpha$ CGRP-specific, we used NK1-R antagonist (GR 82,334) and CGRP-R antagonist (SB 273779) for treatment of a human chondrocyte cell line (C28/I2), which reacts comparably to non-OA-CH to SP and $\alpha \mathrm{CGRP}$ treatment. In both cases, the receptor antagonists were able to reduce the phosphorylation of ERK and AKT, when applied together with SP or $\alpha$ CGRP (Supplementary Figures S5A, B).

Both neuropeptides induced phosphorylation of ERK1/2 in $\mathrm{OA}$ - and in non-OA $\mathrm{CH}$ whereas AKT-phosphorylation was only induced by both neuropeptides in non-OA-CH.

\section{DISCUSSION}

Neurotransmitters of the sensory nervous system can influence the physiology of various cell types of the musculoskeletal system, mainly acting as trophic factors in this context (1416). We have previously reported profound effects of SP and $\alpha$ CGRP on osteoblasts, macrophages and osteoclasts $(5,6)$. 


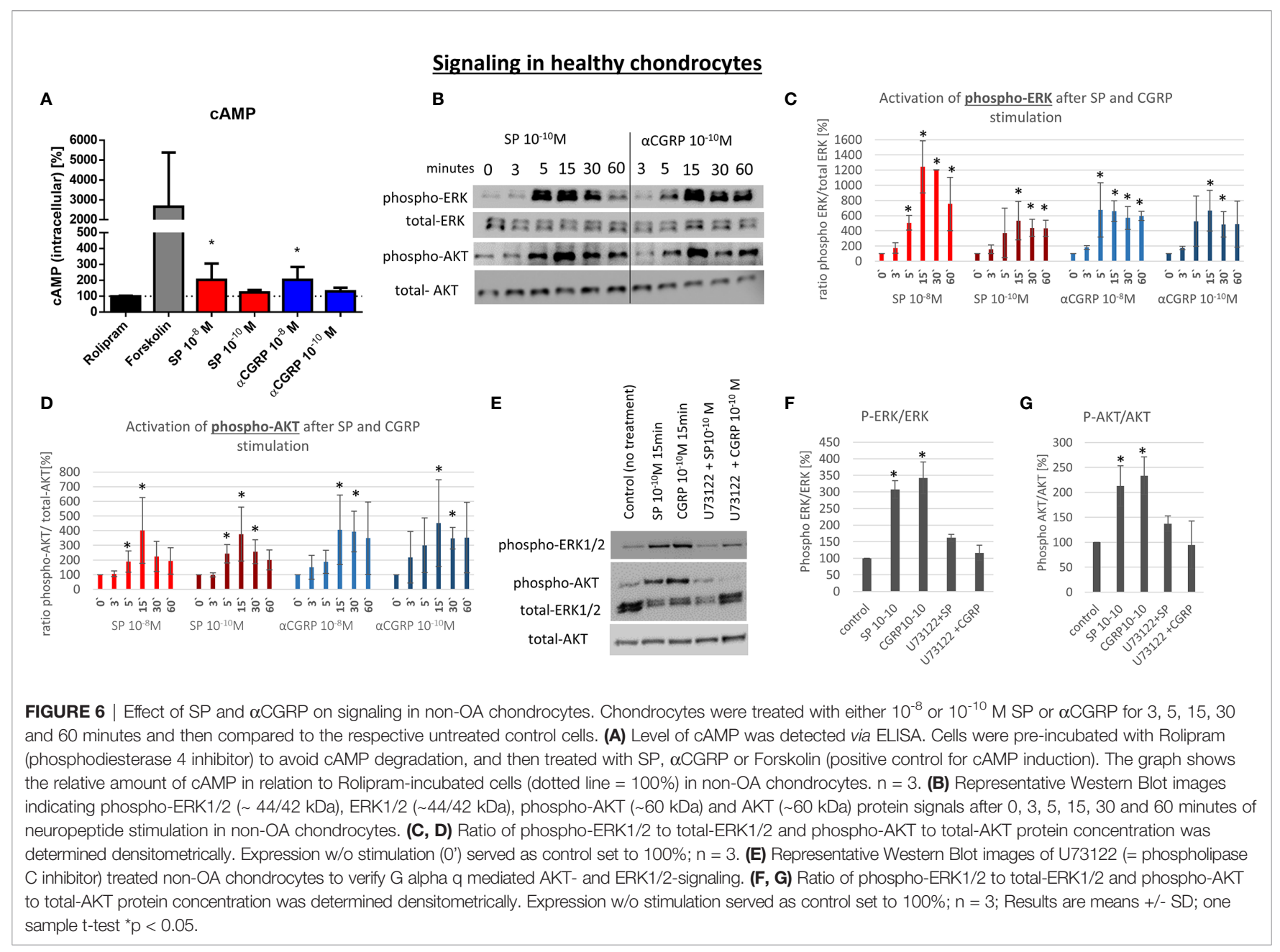

Treatment of bone marrow derived macrophages from tachykinin-1 knockout mice (no SP) with SP reduced proliferation and caspase 3/7 activity, while affecting osteoblast proliferation and caspase activity in a time-dependent manner (5). Mature adult $\alpha$ CGRP-deficient mice showed an increase of dysfunctional mature osteoblasts (6). Studies analyzing effects of the sensory neurotransmitters SP and $\alpha$ CGRP on chondrocyte metabolism are rare. Hence, we focused in our study on catabolic/anabolic cellular effects of the neuropeptides and compared their influence on chondrocytes from OA patients and non-OA, healthy donors. This may help to better assess the mode of SP and $\alpha$ CGRP effects in OA knee joints, where articular cartilage mostly consists of both osteoarthritic and healthy appearing regions (17).

We detected no difference in the concentration/expression of SP or NK1-R between OA- and non-OA chondrocytes. Taken together with the results of Im et al., who observed a significant increase of SP in the synovial fluid of OA patients (18), it can be assumed that chondrocytes are not responsible for the increased SP level in synovial fluid during OA progression. Rather, other cell types, such as synovial fibroblasts, are considered to be a major source of SP production and release (19). For $\alpha$ CGRP, it has been shown that the expression $(20,21)$ and release $(22,23)$ is increased in afferents from rat and murine joints affected by OA. The amount of $\alpha$ CGRP released from human OA chondrocytes was increased by trend in our study in comparison to chondrocytes from healthy cartilage. At the same time, protein expression of RAMP-1, the $\alpha$ CGRP specific subunit component of the $\alpha \mathrm{CGRP}$ receptor, was significantly decreased in $\mathrm{OA}$ chondrocytes in comparison with non-OA chondrocytes, indicating that there may be an increased sensitivity of chondrocytes specifically to $\alpha$ CGRP during OA pathology.

In the present study, we observed that both, SP and $\alpha$ CGRP negatively affect the viability of chondrocytes, however at specific time points only, with SP decreasing viability of OAchondrocytes and $\alpha$ CGRP decreasing viability of healthy chondrocytes which is reflected in increased apoptosis after SP stimulation and decreased proliferation after $\alpha$ CGRP treatment. These data emphasize the critical effects deriving from the environment of the cells. We demonstrated dose- and timedependent negative effects of $\alpha$ CGRP on OA chondrocytes in proliferation, apoptosis and senescence, whereas in non-OA chondrocytes, the influence of $\alpha \mathrm{CGRP}$ was mainly protective, with an anti-apoptotic and anti-senescence effect (reduced p16 gene expression). This is in line with previous results from our 


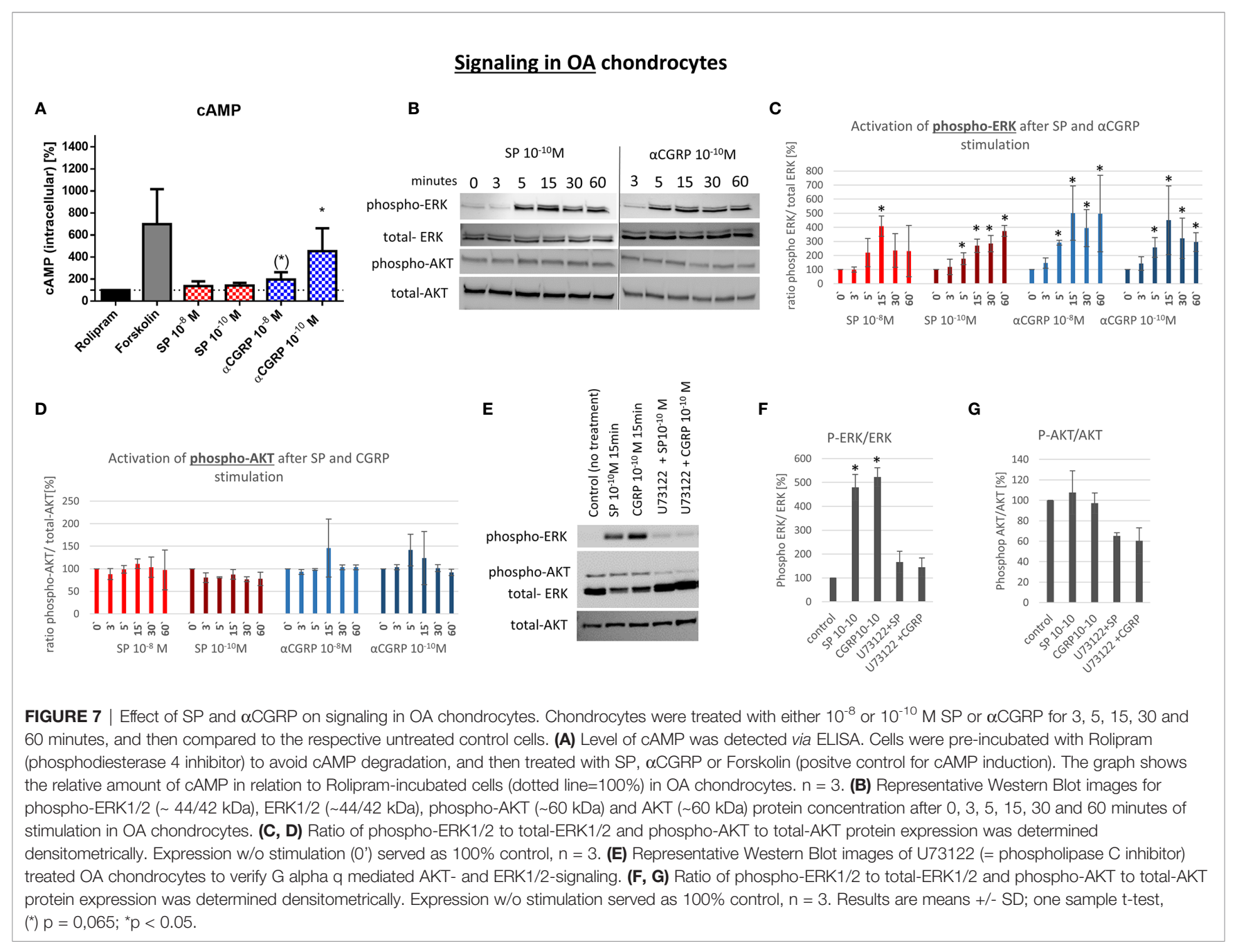

group for murine osteoblasts and osteoclasts (6). $\alpha$ CGRP applied in a high dose for 7 days had anti-proliferative effects on OA- and non-OA chondrocytes in our experiments, whereas a lower dose did not influence the growth rate in any of the groups. In contrast, a high SP dose decreased the proliferation of OA chondrocytes after a short period of treatment (3 days). This contrasts with the work of Opolka et al. who reported a dosedependent increase in the proliferation of primary costal chondrocytes from newborn mice with SP (10), indicating that the effects of SP and $\alpha$ CGRP depend on the type and physiological status of the target cells. $\alpha$ CGRP effectively enhanced primary rat osteoblast proliferation (24), but exerted anti-proliferative effects in aortic and pulmonary artery smooth muscle cells (25). SP treatment stimulates proliferation in bone marrow stromal cells (15) and in fibroblasts during tendon repair $(26,27)$. Growth inhibiting effects of SP, as we have observed for OA chondrocytes, have also been reported, for example in hair follicle cells (28). Clinical therapeutic approaches that use autologous chondrocyte implantation for focal cartilage lesion repair demonstrated that the adhesion of chondrocytes is an essential step for successful regeneration. Based on our results, and considering those of others, we speculate that altered SP or $\alpha$ CGRP levels in synovial fluid or joint tissues could delay or impair engraftment of healthy chondrocytes subsequent to implantation. We observed reduced gene expression of integrin $\alpha 11$ in OA-chondrocytes after neuropeptide treatment whereas gene expression in chondrocytes from healthy donors was not affected. The difference between OA- and non-OA chondrocytes in terms of adhesion after SP or $\alpha$ CGRP treatment may thus either result from reduced expression of integrins or a switch to expression of different subsets of integrins in chondrocytes as degeneration progresses (29).

The phenotype of chondrocytes can be defined by analyzing specific markers. The variability of the expression of these markers is age and health status related, but can also be influenced by growth factors or SP or $\alpha$ CGRP. For SP, it is reported that it can accelerate terminal differentiation of chondrocytes (30) and it seems to be essential for cartilage homeostasis because it participates in transduction of mechanical cues through the NKR-1 (3, 31, 32). In contrast, 


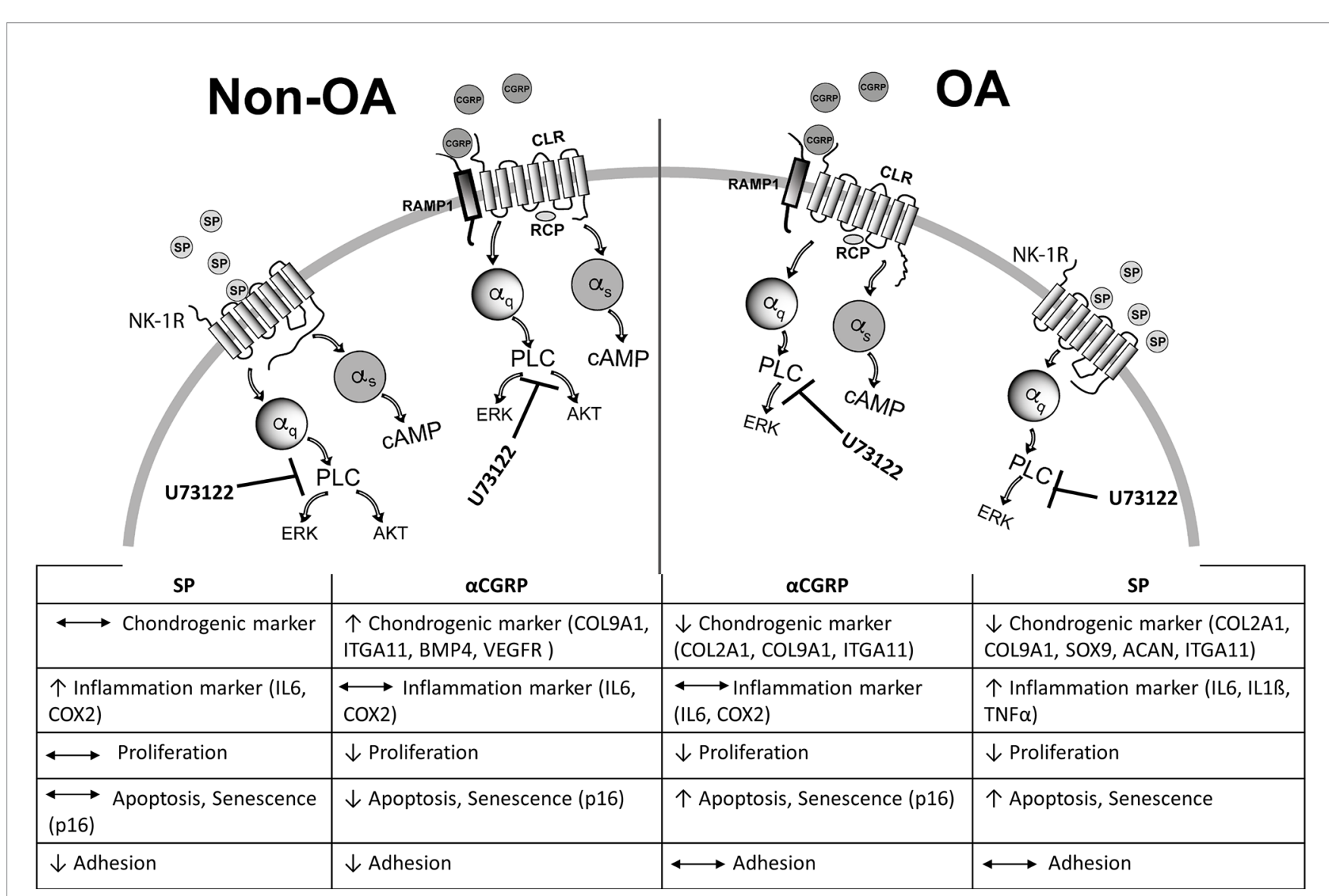

FIGURE 8 | Schematic summary of SP and $\alpha$ CGRP metabolic effects and activated signaling pathways in healthy and OA chondrocytes. Binding of SP and $\alpha$ CGRP to their respective receptors results in different physiological effects in $\mathrm{OA}$ - and healthy chondrocytes presumably due to activation of partly different signalling pathways in both chondrocyte groups.

knowledge of the effects of $\alpha$ CGRP on chondrocytes and cartilage biology is scarce. We demonstrated a stimulating and positive impact of $\alpha$ CGRP on chondrogenic marker gene expression (COL9A1, ITGA11, BMP-4) in healthy chondrocytes, whereas in OA chondrocytes, the same $\alpha$ CGRP concentration inhibited gene expression of these markers (COL2A1, COL9A1, ITGA11). We observed a strong inhibition of COL2A1 and COL9A1 gene expression when OA chondrocytes were treated with a low SP concentration and downregulation of SOX9, ACAN, ITGA11 after treatment with both SP concentrations. These observations are in line with recent studies showing that SP plays an important role in the process of OA cartilage degeneration $(33,34)$ and may imply that an increased SP level in the joints can accelerate destructive processes during OA pathology or hamper healing processes. We have previously reported that in a murine destabilizationinduced osteoarthritis model the cartilage degradation was delayed in SP-deficient mice (35), underlining our observations of the catabolic influence of SP on OA chondrocytes in the present study.

In addition, expression of inflammation-related genes was increased in OA chondrocytes treated with SP, whereas non-OA chondrocytes showed little response. Pro-inflammatory cytokines, commonly secreted by immune cells, are the main driver of the low-grade inflammation during OA pathology (36). However, expression of the pro-inflammatory cytokines IL1 $\beta$ and TNF $\alpha$, classically secreted in early OA (37), was significantly increased in OA chondrocytes after SP treatment, indicating a critical role of SP in the inflammatory response of chondrocytes. Also for other musculoskeletal tissues, such as tendons, it has been shown that SP can promote inflammation (38). We demonstrated a dose-dependent effect of SP on inflammation markers indicating the possibility that while a low SP dose does not critically alter the inflammation process in the joint, higher doses of SP have destructive effects on joint tissues by reinforcing the inflammatory milieu. It is notable that $\alpha$ CGRP did not affect the expression of any tested inflammation marker in both chondrocyte groups, indicating no direct influence of $\alpha$ CGRP on inflammatory processes via chondrocytes in the joints of OA patients. Nevertheless, it is likely that the peripheral actions of $\alpha$ CGRP in the joints do contribute to inflammation and also to joint afferent sensitization (39), as $\alpha$ CGRP is also linked to inflammatory pain by attenuating responses in $\alpha$ CGRP knockout mice in a number of pain models $(40,41)$. The increase of the $\alpha$ CGRP concentration in OA joints, as reported by Tankano et al., does not drive specifically chondrocytes to intensify the 
inflammatory process but would, through other cell types such as synovial fibroblasts, trigger catabolic processes in OA joints (42).

The receptors for SP and $\alpha$ CGRP are classical G proteincoupled receptors (GPCR). These receptors can activate at least three types of G-proteins: Gs, which activates adenylyl cyclase (AC)/protein kinase A (PKA), the Gq/G11 family, which activates phospholipase C (PLC)/protein kinase C (PKC), and Gi, which can inhibit adenylyl cyclase (43). Activation of Gos and cAMP exert anti-inflammatory effects, whereas activation of $\mathrm{G} \alpha \mathrm{q}$ and PLC affect the receptor-stimulated mitogen-activated-proteinkinase (MAPK) pathway resulting in pro-inflammatory stimuli $(44,45)$. In our study, OA- and non-OA chondrocytes increased the production of the second messenger cAMP after $\alpha$ CGRP binding to its receptor, confirming the data of Edoff et al., who reported similar results when rat chondrocytes were treated with $\alpha$ CGRP (46). $\alpha$ CGRP is able to increase cAMP in a concentrationdependent manner in several cell types, as was demonstrated in neonatal dorsal root ganglion (DRG) neurons (47). Moreover, treatment with a high dose of SP increased the cAMP level in nonOA chondrocytes similar to treatment with a high dose of $\alpha$ CGRP. In contrast, SP did not alter the cAMP response in OA chondrocytes, indicating no anti-inflammatory signaling effects for those cells via SP. Interestingly, in OA chondrocytes, a low dose of $\alpha$ CGRP induced cAMP synthesis, whereas in non-OA chondrocytes, a high dose of $\alpha$ CGRP was needed to increase the cAMP level, emphasizing the different sensitivity of OA- and healthy chondrocytes to exogenous stimuli. Beside the antiinflammatory effects of $\alpha$ CGRP and, in part of SP, we suggest that the pro-inflammatory and catabolic effects may be mediated via $\mathrm{Gq}$ and PLC activation given the observed ERK and AKT pathway activation, which could be blocked by the specific PLC inhibitor U73122. In healthy chondrocytes, ERK and AKT were phosphorylated 5-15 minutes after SP or $\alpha$ CGRP addition. Of note, in OA chondrocytes binding of SP and $\alpha$ CGRP to their receptors only induced phosphorylation of ERK, but not of AKT. Conflicting data concerning AKT signaling in chondrocytes were reported previously. Whereas Appleton et al. postulated articular cartilage degradation after AKT phosphorylation via increased MMP-13 expression (48), others reported that AKT signaling promotes matrix synthesis and the survival of chondrocytes, and that a constitutively active AKT signaling pathway in human articular chondrocytes resulted in increased proteoglycan synthesis and Sox9 expression $(49,50)$. AKT phosphorylation was not changed in OA chondrocytes after SP and $\alpha$ CGRP treatment, again underlining different sensitivity of OA- and non-OA chondrocytes to external stimuli. We therefore suggest that induction in non-OA chondrocytes may depend on the health status or the younger age of the chondrocyte donors. As phosphorylation of ERK was induced in both chondrocyte groups with both neuropeptides, we postulate a common signaling mechanism by SP and $\alpha$ CGRP, which is independent of disease status or donor age. It is well known that MAPK signaling pathways, which include ERK, play an important role in the pathogenesis of $\mathrm{OA}$ and that the expression of MMPs in chondrocytes is associated with the activation of MAPK signaling $(51,52)$.

We want to point out that donors of OA- and healthy chondrocytes were of significant different ages indicating that typical age-related comorbidities are lacking in the donors of the healthy chondrocytes. This shall be kept in mind especially when considering the senescence and apoptosis data which might not only be related to OA but also to the different age of the donors.

\section{CONCLUSION}

We suggest that there is a pro-inflammatory and destructive effect through active ERK signaling induced by SP and $\alpha$ CGRP in both healthy and OA articular chondrocytes. This catabolic response could be counteracted by an anti-inflammatory and anabolic effect exerted by the cAMP response, particularly as induced by $\alpha$ CGRP addition in non-OA chondrocytes. We suggest that there is a balance between the more catabolic effects of SP and the anabolic effects of $\alpha$ CGRP in healthy joints. However, this balance may be disturbed by the chronic inflammatory milieu in joints of OA patients and shifted toward a catabolic response to SP. Therapeutic approaches that induce a change in one neuropeptide, either SP or CGRP, should therefore include monitoring of the other neuropeptide.

Moreover, we conclude that a change in the involved alpha subunits of the corresponding G-protein-coupled-receptors after binding SP and $\alpha$ CGRP might play a central role in mediating the trophic effects of these neuropeptides on healthy versus OA chondrocytes. For healthy chondrocytes, we assume that alpha q, as well as alpha s subunits, can assemble to the receptors for SP or $\alpha C G R P$ thereby affecting downstream signaling pathways, whereas in OA chondrocytes, SP is unable to induce a Gos assembly to the NK1-R. A schematic overview summarizes signaling events that could occur based on the effects we detected for SP and $\alpha$ CGRP treatment of healthy versus OA chondrocytes (Figure 8).

\section{DATA AVAILABILITY STATEMENT}

The raw data supporting the conclusions of this article will be made available by the authors, without undue reservation.

\section{ETHICS STATEMENT}

The studies involving human participants were reviewed and approved by Ethics committee at the University of Regensburg, Az: 14-101-0189, email: ethikkommission@klinik.ukr.de. The patients/participants provided their written informed consent to participate in this study.

\section{AUTHOR CONTRIBUTIONS}

SS: Conception and design, analysis and interpretation of the data, drafting of the original article. AE: Seahorse metabolic analysis, review and editing. RB: Graphic support, review and editing. MK: sample acquisition, review and editing. BJ: sample acquisition, review and editing. SG: Conception and design, review and editing, project administration, funding acquisition. All authors contributed to the article and approved the submitted version. 


\section{FUNDING}

This work was funded by a grant from the German Research Foundation (DFG) assigned to SG [GR 1301/18-1].

\section{ACKNOWLEDGMENTS}

We thank Anja Pasoldt and Mandy Vogel for their excellent technical assistance. We also are indepted to Sylvia Müller and Thomas Kamradt, Dept. of Immunology, University of Jena, for

\section{REFERENCES}

1. Loeser RF, Goldring SR, Scanzello CR, Goldring MB. Osteoarthritis: A Disease of the Joint as an Organ. Arthritis Rheumatism (2012) 64:1697707. doi: $10.1002 /$ art.34453

2. Martel-Pelletier J, Barr AJ, Cicuttini FM, Conaghan PG, Cooper C, Goldring MB, et al. Osteoarthritis. Nat Rev Dis Primers (2016) 2:16072. doi: 10.1038/ nrdp. 2016.72

3. Grassel SG. The Role of Peripheral Nerve Fibers and Their Neurotransmitters in Cartilage and Bone Physiology and Pathophysiology. Arthritis Res Ther (2014) 16:485. doi: 10.1186/s13075-014-0485-1

4. Grassel S, Muschter D. Peripheral Nerve Fibers and Their Neurotransmitters in Osteoarthritis Pathology. Int J Mol Sci (2017) 18(5):931. doi: 10.3390/ ijms 18050931

5. Niedermair T, Schirner S, Seebroker R, Straub RH, Grassel S. Substance P Modulates Bone Remodeling Properties of Murine Osteoblasts and Osteoclasts. Sci Rep (2018) 8:9199. doi: 10.1038/s41598-018-27432-y

6. Niedermair T, Schirner S, Lasheras MG, Straub RH, Grassel S. Absence of Alpha-Calcitonin Gene-Related Peptide Modulates Bone Remodeling Properties of Murine Osteoblasts and Osteoclasts in an AgeDependent Way. Mech Ageing Dev (2020) 189:111265. doi: 10.1016/ j.mad.2020.111265

7. Buma $P$, Verschuren $C$, Versleyen D, van der Kraan $P$, Oestreicher $A B$. Calcitonin Gene-Related Peptide, Substance P and GAP-43/B-50 Immunoreactivity in the Normal and Arthrotic Knee Joint of the Mouse. Histochemistry (1992) 98:327-39. doi: 10.1007/BF00270017

8. Suri S, Gill SE, Massena de Camin S, Wilson D, McWilliams DF, Walsh DA. Neurovascular Invasion at the Osteochondral Junction and in Osteophytes in Osteoarthritis. Ann Rheumatic Dis (2007) 66:1423-8. doi: 10.1136/ ard.2006.063354

9. Oliva F, Tarantino U, Maffulli N. Immunohistochemical Localization of Calcitonin Gene-Related Peptide and Substance P in the Rat Knee Cartilage at Birth. Physiol Res (2005) 54:549-56.

10. Opolka A, Straub RH, Pasoldt A, Grifka J, Grassel S. Substance P and Norepinephrine Modulate Murine Chondrocyte Proliferation and Apoptosis. Arthritis Rheumatism (2012) 64:729-39. doi: 10.1002/art.33449

11. Leyh M, Seitz A, Durselen L, Springorum HR, Angele P, Ignatius A, et al. Osteoarthritic Cartilage Explants Affect Extracellular Matrix Production and Composition in Cocultured Bone Marrow-Derived Mesenchymal Stem Cells and Articular Chondrocytes. Stem Cell Res Ther (2014) 5:77. doi: 10.1186/scrt466

12. Lorenz J, Schafer N, Bauer R, Jenei-Lanzl Z, Springorum RH, Grassel S. Norepinephrine Modulates Osteoarthritic Chondrocyte Metabolism and Inflammatory Responses. Osteoarthritis Cartilage/OARS Osteoarthritis Res Soc (2016) 24:325-34. doi: 10.1016/j.joca.2015.08.007

13. Leyh M, Seitz A, Durselen L, Schaumburger J, Ignatius A, Grifka J, et al. Subchondral Bone Influences Chondrogenic Differentiation and Collagen Production of Human Bone Marrow-Derived Mesenchymal Stem Cells and Articular Chondrocytes. Arthritis Res Ther (2014) 16:453. doi: 10.1186/ s13075-014-0453-9

14. Goto T, Nakao K, Gunjigake KK, Kido MA, Kobayashi S, Tanaka T. Substance P Stimulates Late-Stage Rat Osteoblastic Bone Formation Through Neurokinin-1 Receptors. Neuropeptides (2007) 41:25-31. doi: 10.1016/ j.npep.2006.11.002

15. Wang L, Zhao R, Shi X, Wei T, Halloran BP, Clark D, et al. Substance P Stimulates Bone Marrow Stromal Cell Osteogenic Activity, Osteoclast giving us the opportunity to use their Seahorse analyzer and helped with their technical assistance.

\section{SUPPLEMENTARY MATERIAL}

The Supplementary Material for this article can be found online at: https://www.frontiersin.org/articles/10.3389/fimmu.2021. 722884/full\#supplementary-material

Differentiation, and Resorption Activity In Vitro. Bone (2009) 45:309-20. doi: 10.1016/j.bone.2009.04.203

16. Lerner UH, Persson E. Osteotropic Effects by the Neuropeptides Calcitonin Gene-Related Peptide, Substance $\mathrm{P}$ and Vasoactive Intestinal Peptide. J Musculoskeletal Neuronal Interact (2008) 8:154-65.

17. Cooke ME, Lawless BM, Jones SW, Grover LM. Matrix Degradation in Osteoarthritis Primes the Superficial Region of Cartilage for Mechanical Damage. Acta Biomater (2018) 78:320-8. doi: 10.1016/j.actbio.2018.07.037

18. Im HJ, Li X, Muddasani P, Kim GH, Davis F, Rangan J, et al. Basic Fibroblast Growth Factor Accelerates Matrix Degradation via a Neuro-Endocrine Pathway in Human Adult Articular Chondrocytes. J Cell Physiol (2008) 215:452-63. doi: 10.1002/jcp.21317

19. Inoue H, Shimoyama $Y$, Hirabayashi K, Kajigaya H, Yamamoto S, Oda H, et al. Production of Neuropeptide Substance P by Synovial Fibroblasts From Patients With Rheumatoid Arthritis and Osteoarthritis. Neurosci Lett (2001) 303:149-52. doi: 10.1016/S0304-3940(01)01713-X

20. Fernihough J, Gentry C, Bevan S, Winter J. Regulation of Calcitonin GeneRelated Peptide and TRPV1 in a Rat Model of Osteoarthritis. Neurosci Lett (2005) 388:75-80. doi: 10.1016/j.neulet.2005.06.044

21. Ferreira-Gomes J, Adaes S, Sarkander J, Castro-Lopes JM. Phenotypic Alterations of Neurons That Innervate Osteoarthritic Joints in Rats. Arthritis Rheumatism (2010) 62:3677-85. doi: 10.1002/art.27713

22. Puttfarcken PS, Han P, Joshi SK, et al. A-995662 [(R)-8-(4-Methyl-5-(4(Trifluoromethyl)Phenyl)Oxazol-2-Ylamino)-1,2,3,4-Tetrahydr Onaphthalen-2-Ol], a Novel, Selective TRPV1 Receptor Antagonist, Reduces Spinal Release of Glutamate and CGRP in a Rat Knee Joint Pain Model. Pain (2010) 150:319-26. doi: 10.1016/j.pain.2010.05.015

23. Ogbonna AC, Clark AK, Gentry C, Hobbs C, Malcangio M. Pain-Like Behaviour and Spinal Changes in the Monosodium Iodoacetate Model of Osteoarthritis in C57Bl/6 Mice. Eur J Pain (2013) 17:514-26. doi: 10.1002/ j.1532-2149.2012.00223.x

24. Han N, Zhang DY, Wang TB, Zhang PX, Jiang BG. Calcitonin Gene-Related Peptide Induces Proliferation and Monocyte Chemoattractant Protein-1 Expression via Extracellular Signal-Regulated Kinase Activation in Rat Osteoblasts. Chin Med J (2010) 123:1748-53.

25. Chattergoon NN, D’Souza FM, Deng W, Chen H, Hyman AL, Kadowitz PJ, et al. Antiproliferative Effects of Calcitonin Gene-Related Peptide in Aortic and Pulmonary Artery Smooth Muscle Cells. Am J Physiol Lung Cell Mol Physiol (2005) 288:L202-11. doi: 10.1152/ajplung.00064.2004

26. Steyaert A, Burssens P, Forsyth R, Vanderstraeten G. Qualitative Analysis of Substance P, NK1-Receptor and Nerve Ingrowth in Substance P-Treated Ruptured Rat Achilles Tendon. Acta Orthopaedica Belgica (2010) 76:387-95.

27. Burssens P, Steyaert A, Forsyth R, van Ovost EJ, Depaepe Y, Verdonk R. Exogenously Administered Substance P and Neutral Endopeptidase Inhibitors Stimulate Fibroblast Proliferation, Angiogenesis and Collagen Organization During Achilles Tendon Healing. Foot Ankle Int (2005) 26:832-9. doi: 10.1177/107110070502601008

28. Liu N, Wang LH, Guo LL, Wang GQ, Zhou XP, Jiang Y, et al. Chronic Restraint Stress Inhibits Hair Growth via Substance P Mediated by Reactive Oxygen Species in Mice. PloS One (2013) 8:e61574. doi: 10.1371/journal. pone.0061574

29. Almonte-Becerril M, Costell M, Kouri JB. Changes in the Integrins Expression are Related With the Osteoarthritis Severity in an Experimental Animal Model in Rats. J Orthopaedic Res: Off Publ Orthopaedic Res Soc (2014) 32:1161-6. doi: 10.1002/jor.22649 
30. Li FX, Xu F, Lin X, Wu F, Zhong JY, Wang Y, et al. The Role of Substance P in the Regulation of Bone and Cartilage Metabolic Activity. Front Endocrinol (2020) 11:77. doi: 10.3389/fendo.2020.00077

31. Howard MR, Millward-Sadler SJ, Vasilliou AS, Salter DM, Quinn JP. Mechanical Stimulation Induces Preprotachykinin Gene Expression in Osteoarthritic Chondrocytes Which is Correlated With Modulation of the Transcription Factor Neuron Restrictive Silence Factor. Neuropeptides (2008) 42:681-6. doi: 10.1016/j.npep.2008.09.004

32. Millward-Sadler SJ, Wright MO, Davies LW, Nuki G, Salter DM. Mechanotransduction via Integrins and Interleukin-4 Results in Altered Aggrecan and Matrix Metalloproteinase 3 Gene Expression in Normal, But Not Osteoarthritic, Human Articular Chondrocytes. Arthritis Rheumatism (2000) 43:2091-9. doi: 10.1002/1529-0131(200009)43:9<2091::AIDANR21>3.0.CO;2-C

33. Grimsholm O, Rantapaa-Dahlqvist S, Forsgren S. Levels of Gastrin-Releasing Peptide and Substance P in Synovial Fluid and Serum Correlate With Levels of Cytokines in Rheumatoid Arthritis. Arthritis Res Ther (2005) 7:R416-26. doi: $10.1186 /$ ar 1503

34. Pritchett JW. Substance P Level in Synovial Fluid may Predict Pain Relief After Knee Replacement. J Bone Joint Surg Br Volume (1997) 79:114-6. doi: 10.1302/0301-620X.79B1.0790114

35. Muschter D, Fleischhauer L, Taheri S, Schilling AF, Clausen-Schaumann H, Grassel S. Sensory Neuropeptides are Required for Bone and Cartilage Homeostasis in a Murine Destabilization-Induced Osteoarthritis Model. Bone (2020) 133:115181. doi: 10.1016/j.bone.2019.115181

36. Sokolove J, Lepus CM. Role of Inflammation in the Pathogenesis of Osteoarthritis: Latest Findings and Interpretations. Ther $A d v$ Musculoskeletal Dis (2013) 5:77-94. doi: 10.1177/1759720X12467868

37. Min S, Wang C, Lu W, Xu Z, Shi D, Chen D, et al. Serum Levels of the Bone Turnover Markers Dickkopf-1, Osteoprotegerin, and TNF-Alpha in Knee Osteoarthritis Patients. Clin Rheumatol (2017) 36:2351-8. doi: 10.1007/ s10067-017-3690-x

38. Tuzmen C, Verdelis K, Weiss L, Campbell P. Crosstalk Between Substance P and Calcitonin Gene-Related Peptide During Heterotopic Ossification in Murine Achilles Tendon. J Orthopaedic Res: Off Publ Orthopaedic Res Soc (2018) 36:1444-55. doi: 10.1002/jor.23833

39. Walsh DA, Mapp PI, Kelly S. Calcitonin Gene-Related Peptide in the Joint: Contributions to Pain and Inflammation. Br J Clin Pharmacol (2015) 80:96578. doi: 10.1111/bcp.12669

40. Salmon AM, Damaj MI, Marubio LM, Epping-Jordan MP, Merlo-Pich E, Changeux JP. Altered Neuroadaptation in Opiate Dependence and Neurogenic Inflammatory Nociception in Alpha CGRP-Deficient Mice. Nat Neurosci (2001) 4:357-8. doi: 10.1038/86001

41. Zhang L, Hoff AO, Wimalawansa SJ, Cote GJ, Gagel RF, Westlund KN. Arthritic Calcitonin/Alpha Calcitonin Gene-Related Peptide Knockout Mice Have Reduced Nociceptive Hypersensitivity. Pain (2001) 89:265-73. doi: 10.1016/S0304-3959(00)00378-X

42. Takano S, Uchida K, Inoue G, Minatani A, Miyagi M, Aikawa J, et al. Increase and Regulation of Synovial Calcitonin Gene-Related Peptide Expression in Patients With Painful Knee Osteoarthritis. J Pain Res (2017) 10:1099-104. doi: 10.2147/JPR.S135939

43. Gether U. Uncovering Molecular Mechanisms Involved in Activation of G Protein-Coupled Receptors. Endocri Rev (2000) 21:90-113. doi: 10.1210/ edrv.21.1.0390
44. Montrose-Rafizadeh C, Avdonin P, Garant MJ, Rodgers BD, Kole S, Yang H, et al. Pancreatic Glucagon-Like Peptide-1 Receptor Couples to Multiple G Proteins and Activates Mitogen-Activated Protein Kinase Pathways in Chinese Hamster Ovary Cells. Endocrinology (1999) 140:1132-40. doi: 10.1210/endo.140.3.6550

45. Magocsi M, Vizi ES, Selmeczy Z, Brozik A, Szelenyi J. Multiple G-ProteinCoupling Specificity of Beta-Adrenoceptor in Macrophages. Immunology (2007) 122:503-13. doi: 10.1111/j.1365-2567.2007.02658.x

46. Edoff K, Hildebrand C. Neuropeptide Effects on Rat Chondrocytes and Perichondrial Cells In Vitro. Neuropeptides (2003) 37:316-8. doi: 10.1016/ j.npep.2003.09.001

47. Anderson LE, Seybold VS. Calcitonin Gene-Related Peptide Regulates Gene Transcription in Primary Afferent Neurons. J Neurochem (2004) 91:1417-29. doi: 10.1111/j.1471-4159.2004.02833.x

48. Appleton CT, Usmani SE, Mort JS, Beier F. Rho/ROCK and MEK/ERK Activation by Transforming Growth Factor-Alpha Induces Articular Cartilage Degradation. Lab Invest; J Tech Methods Pathol (2010) 90:20-30. doi: 10.1038/ labinvest.2009.111

49. Yin W, Park JI, Loeser RF. Oxidative Stress Inhibits Insulin-Like Growth Factor-I Induction of Chondrocyte Proteoglycan Synthesis Through Differential Regulation of Phosphatidylinositol 3-Kinase-Akt and MEK-ERK MAPK Signaling Pathways. J Biol Chem (2009) 284:31972-81. doi: 10.1074/ jbc.M109.056838

50. Cravero JD, Carlson CS, Im HJ, Yammani RR, Long D, Loeser RF. Increased Expression of the Akt/PKB Inhibitor TRB3 in Osteoarthritic Chondrocytes Inhibits Insulin-Like Growth Factor 1-Mediated Cell Survival and Proteoglycan Synthesis. Arthritis Rheumatism (2009) 60:492-500. doi: 10.1002/art.24225

51. Clancy R, Rediske J, Koehne C, Stoyanovsky D, Amin A, Attur M, et al. Activation of Stress-Activated Protein Kinase in Osteoarthritic Cartilage: Evidence for Nitric Oxide Dependence. Osteoarthritis Cartilage (2001) 9:294-9. doi: 10.1053/joca.2000.0388

52. Fan Z, Soder S, Oehler S, Fundel K, Aigner T. Activation of Interleukin-1 Signaling Cascades in Normal and Osteoarthritic Articular Cartilage. Am J Pathol (2007) 171:938-46. doi: 10.2353/ajpath.2007.061083

Conflict of Interest: The authors declare that the research was conducted in the absence of any commercial or financial relationships that could be construed as a potential conflict of interest.

Publisher's Note: All claims expressed in this article are solely those of the authors and do not necessarily represent those of their affiliated organizations, or those of the publisher, the editors and the reviewers. Any product that may be evaluated in this article, or claim that may be made by its manufacturer, is not guaranteed or endorsed by the publisher.

Copyright (๑ 2021 Stöckl, Eitner, Bauer, König, Johnstone and Grässel. This is an open-access article distributed under the terms of the Creative Commons Attribution License (CC BY). The use, distribution or reproduction in other forums is permitted, provided the original author(s) and the copyright owner(s) are credited and that the original publication in this journal is cited, in accordance with accepted academic practice. No use, distribution or reproduction is permitted which does not comply with these terms. 